\title{
Quantitative analysis of the grain boundary properties in a generalized phase field model for grain growth in anisotropic systems
}

\author{
N. Moelans, B. Blanpain, P. Wollants* \\ Department of Metallurgy and Materials Engineering, \\ Katholieke Universiteit Leuven, Kasteelpark Arenberg 44, 3001 Leuven, Belgium
}

(Dated: February 16, 2008)

\begin{abstract}
A good choice of model formulation and model parameters is one of the most important and difficult aspects in mesoscale modeling and requires a systematic and quantitative analysis. In this article, it is studied for a generalized phase field model, how the model parameters affect the landscape of the free energy density functional, the phase field profiles at the grain boundaries and the corresponding trajectory along the free energy landscape. This analysis results in quantitative relations between the model parameters, on the one hand, and grain boundary energy and mobility as a function of misorientation and grain boundary inclination, on the other hand. Based on these findings, an algorithm is implemented that generates a suitable set of model parameters for given grain boundary energies and mobilities and a prescribed value for the diffuse interface width. The misorientation and inclination dependence of the grain boundary energy are introduced in such a way that the diffuse interface width is constant, resulting in uniform stability and accuracy conditions for the numerical solution. The proposed model formulation and parameter choice allow us to perform quantitative simulations with excellent controllability of the numerical accuracy.
\end{abstract}

PACS numbers: Valid PACS appear here 


\section{INTRODUCTION}

Simulation techniques that describe microstructural evolution on a mesoscale, such as the phase field method, are very important in grain growth studies. When grain boundary energy and mobility are known from experiments, atomistic simulations or theory, mesoscale simulations allow to predict the evolution of the grain size and grain orientation distributions of a given grain structure. They have been extremely valuable in the validation of mean field theories, for instance, for ideal grain growth ${ }^{1-4}$ and grain growth in the presence of second-phase particles ${ }^{5-9}$. Currently, many attention is also given to the evolution of structures in which grain boundary energy and mobility depend on the misorientation between neighboring grains and the grain boundary inclination ${ }^{10,11}$. Mesoscale simulations allow us to distinguish between the effects caused by anisotropy in grain boundary energy and those caused by anisotropy in grain boundary mobility ${ }^{12,13}$. The misorientation dependence of grain boundary properties could also play an important role in the nucleation of abnormal grain and subgrain growth ${ }^{14}$. It is, however, still a great challenge to derive reliable conclusions from mesoscale grain growth simulations.

One of the difficulties in mesoscale modelling is that material properties that follow from processes and interactions on the atomistic scale must be introduced into the model in a phenomenological way. In mesoscale models, the properties of a system are usually described with parameterized expressions and the evolution equations are derived based on thermodynamic principles. The phenomenological parameters are related to material properties relevant for the considered process, such as grain boundary energy and mobility in the case of grain growth. Ideally, the phenomenological description captures the important physics and is free from non-physical side-effects. It is also a good modelling approach to reduce the number of model parameters as far as possible. Still, the choice of the phenomenological expressions and model parameters is somehow arbitrary. There may be different mesoscale description for the same physical processes and phenomena. Furthermore, material properties, like grain boundary energy and mobility, are not always explicit parameters in the phenomenological description; they can be a complicated function of the model parameters. Sometimes, a number of model parameters is also added for numerical or other practical reasons. As a consequence, it is not straightforward to choose a good set of model parameters that reproduces accurately the desired material properties in the simulations. 
In this article, a systematic analysis is made of the effect of each model parameter on the grain boundary properties for a generalized phase field (or continuum field) model for grain growth, recently proposed by the author ${ }^{15}$. The model is based on the phase field formulation for normal grain growth from Chen and Yang ${ }^{16-18}$, in which the different grain orientations are represented by a large set of non-conserved order parameters or phase field variables that are continuous functions of the spacial coordinates and time

$$
\eta_{1}(\mathbf{r}, t), \eta_{2}(\mathbf{r}, t), \eta_{3}(\mathbf{r}, t), \ldots, \eta_{i}(\mathbf{r}, t), \ldots \eta_{p}(\mathbf{r}, t)
$$

Within the grains, the phase field variables have nearly constant values and at the grain boundaries, they change gradually within a narrow region between their values in the neighboring grains. Grain boundaries are thus diffuse and have a finite width. The description assumes a constant molar volume and a system in thermal equilibrium. The movement of the grain boundaries is implicitly given by the temporal evolution of the phase field variables, which follows a time-dependent Ginzburg-Landau equation

$$
\begin{aligned}
\frac{\partial \eta_{i}(\mathbf{r}, t)}{\partial t} & =-L \frac{\delta F\left(\eta_{1}, \eta_{2}, \ldots, \eta_{p}\right)}{\delta \eta_{i}(\mathbf{r}, t)} \\
& =-L\left[\frac{\partial f\left(\eta_{1}, \eta_{2}, \ldots, \eta_{p}\right)}{\partial \eta_{i}}-\kappa \nabla^{2} \eta_{i}\right]
\end{aligned}
$$

with $i=1 \ldots p$ and $L$ a positive kinetic parameter. These equations guarantee a continuous decrease of the total free energy of the system, according to thermodynamic laws. The total free energy of the system $F$ is expressed as a volume integral over the system, which is a functional of the phase field variables and their gradients

$$
F=\int_{V}\left[f\left(\eta_{1}, \eta_{2}, \ldots, \eta_{p}\right)+\frac{\kappa}{2} \sum_{i=1}^{p}\left(\nabla \eta_{i}\right)^{2}\right] \mathrm{d} V
$$

The parameter $\kappa$ is strictly positive, so that gradients of the phase field variables give rise to a positive contribution to the free energy of the system. The homogeneous free energy density $f$ is a landau polynomial of the phase field variables with multiple degenerate minima for the different grain orientations. Anisotropy can be introduced into the model ${ }^{19,20}$ by formulating $\kappa, \gamma$ and $L$ as a function of the local misorientation and grain boundary inclination, which are derived from the local values of the phase field variables and their gradients ${ }^{11,13,21-23}$.

It was derived, for isotropic systems, that the grain boundary energy is proportional with $\sqrt{\kappa}$ and that the grain boundary velocity equals $L \kappa\left(1 / \rho_{1}+1 / \rho_{2}\right)$, with $1 / \rho_{1}+1 / \rho_{2}$ the local 
mean curvature ${ }^{18,24,25}$. The proportionality constant in the grain boundary energy is however given by an integral expression, which is in general too complicated to work out analytically. Moreover, for phase field models, it is important from a numerical point of view to control the shape and width of the phase field profiles in the diffuse grain boundary regions ${ }^{26}$. Mostly, it is assumed ${ }^{20,22,23}$ that the grain boundary width is proportional with $\sqrt{\kappa /(\Delta f)_{\max }}$, where $(\Delta f)_{\max }$ is defined as 'the maximum height of the barrier in the homogeneous free energy density $f$ between two degenerate minima', although it is not specified how this value is determined. A further understanding of the effect of the model parameters and the shape of the free energy functional on grain boundary properties and diffuse interface width, is thus required to choose an appropriate set of model parameters and obtain accurate simulation results. Furthermore, if the role of each model parameter is understood, it might become possible to derive indirectly difficult-to-measure material properties, such as grain boundary energy and mobility by comparing mesoscale simulation images with experimental microscope images.

In section II, the effect of the model parameters on the shape of the free energy landscape and the phase field profiles at an interface in local equilibrium, is studied for two-grain structures and systems with uniform grain boundary properties. The grain boundary energy, mobility and width are calculated as a function of the model parameters. In section III, this analysis is extended to a generalized phase field model for systems with non-uniform grain boundary properties. Furthermore, a procedure for calculating appropriate sets of model parameters is explained. A number of conclusions and future directions are formulated in section IV. Applications of the model are described elsewhere ${ }^{15,27}$. The analysis is performed specifically for phase field models based on the formulation from Chen and Yang ${ }^{16}$. The conclusions may therefore not apply to other phase field models for grain growth, such as the multi-phase field ${ }^{28}$ and vector-valued models ${ }^{29}$. 


\section{INDIVIDUAL GRAIN BOUNDARIES WITH UNIFORM PROPERTIES}

\section{A. Model formulation for systems with uniform grain boundary properties}

Based on previous experience ${ }^{6,8,30}$, we propose a free energy functional

$$
\begin{array}{r}
F=\int_{V} f\left(\eta_{1}, \ldots, \eta_{p}, \nabla \eta_{1}, \ldots, \nabla \eta_{p}\right) \mathrm{d} V \\
=\int_{V}\left[m f_{0}\left(\eta_{1}, \eta_{2}, \ldots, \eta_{p}\right)+\frac{\kappa}{2} \sum_{i=1}^{p}\left(\nabla \eta_{i}\right)^{2}\right] \mathrm{d} V
\end{array}
$$

with

$$
\begin{gathered}
f_{0}\left(\eta_{1}, \eta_{2}, \ldots, \eta_{p}\right)= \\
\sum_{i=1}^{p}\left(\frac{\eta_{i}^{4}}{4}-\frac{\eta_{i}^{2}}{2}\right)+\gamma \sum_{i=1}^{p} \sum_{j>i}^{p} \eta_{i}^{2} \eta_{j}^{2}+\frac{1}{4},
\end{gathered}
$$

which is slightly different from the original ${ }^{16}$. The number of parameters is reduced with 1 without loss of generality. Moreover, this formulation results in simpler expressions for the grain boundary properties as a function of model parameters. The minima of the homogeneous free energy are located at

$$
\begin{gathered}
\left(\eta_{1}, \eta_{2}, \ldots, \eta_{p}\right)= \\
( \pm 1,0, \ldots, 0),(0, \pm 1,0, \ldots, 0), \ldots,(0, \ldots, 0, \pm 1)
\end{gathered}
$$

where $f_{0}=f_{0, \min }=0$. Consequently, within grains, the free energy density $f$ equals zero and, within the diffuse grain boundary regions, it gives the local contribution to the excess free energy from grain boundaries. Equation (3) gives therefore the total grain boundary energy present in the system. The evolution of the phase field variables is given by equations (1) with $f=f_{0}$.

\section{B. Equations for a flat interface in equilibrium}

To derive equations for the phase field profiles and grain boundary energy of a flat interface in local equilibrium, the two-grain structure shown in figure 1a with grain orientations $i$ and $j$ and a flat interface at $\mathrm{x}=0$, is considered. The grain boundary properties are 
a)
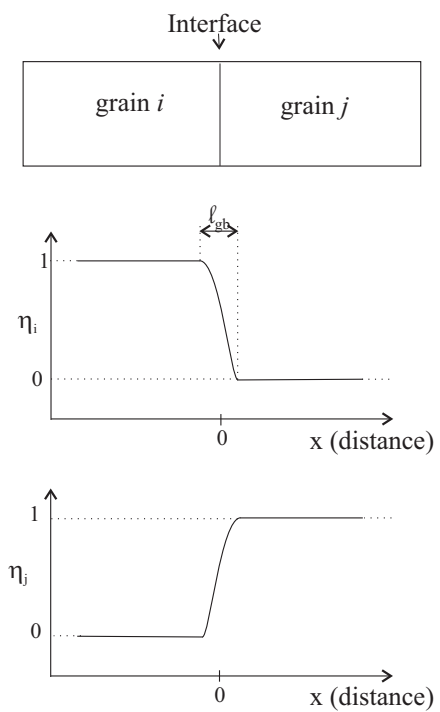

b)
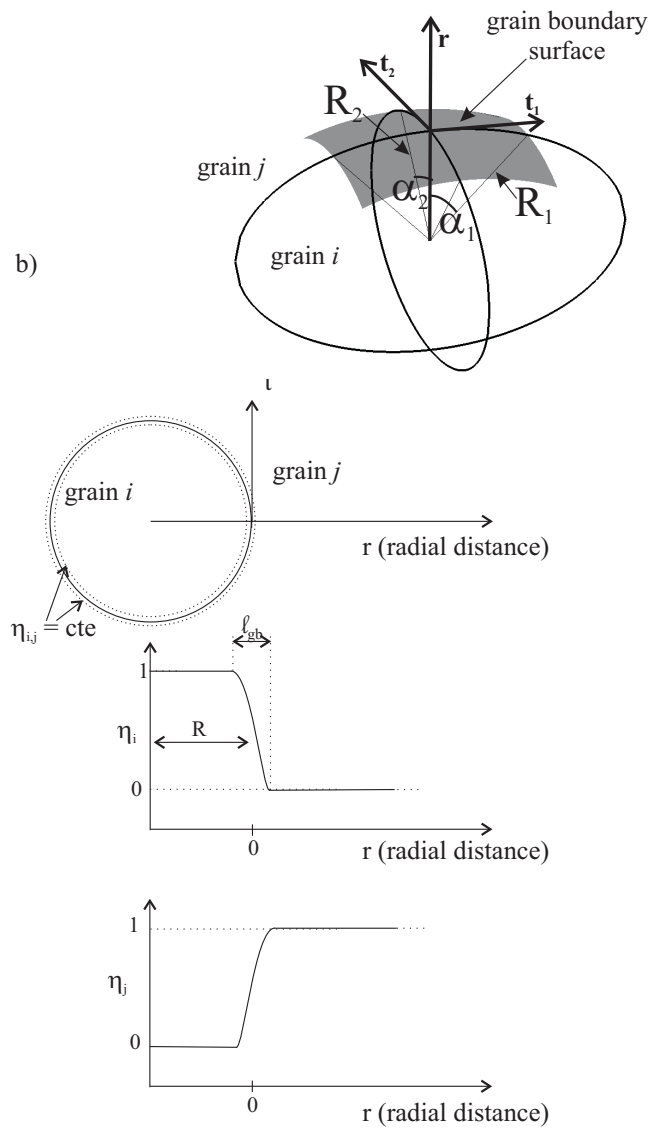

FIG. 1: a) Phase field representation by means of 2 phase field variables $\eta_{i}$ and $\eta_{j}$ of a flat grain boundary between two grains with orientations $i$ and $j . \ell_{g b}$ is a measure for the width of the diffuse grain boundary region where the phase field variables change between their values in the neighboring grains. b) Curved grain boundary surface with mean curvatures $1 / R_{1}$ and $1 / R_{2}$ and profiles of the phase field variables along the normal to the grain boundary surface, for a grain with orientation $i$ embedded in a grain with orientation $j$. A curvilinear coordinate system $\left\{\mathbf{r}, \mathbf{t}_{1}, \mathbf{t}_{2}\right\}$ is indicated, with $\mathbf{r}$ normal to the surface and pointing outwards and $\mathbf{t}_{1}$ and $\mathbf{t}_{2}$ tangential to the surface. The angles $\alpha_{1}$ and $\alpha_{2}$ are measured with respect to the $\mathbf{r}$-axis in the $\mathbf{r}-\mathbf{t}_{1}$ and $\mathbf{r}$ - $\mathbf{t}_{2}$ plane, respectively.

characterized by parameters $m, \gamma_{i, j}$ and $\kappa_{i, j}$ (the parameter nomenclature for non-uniform systems is already applied, in which $\gamma_{i, j}$ and $\kappa_{i, j}$ may have different values for different grain boundaries). Following the approach of Cahn and Hilliard ${ }^{31}$ and using the free energy functional (3-4), the specific grain boundary energy of the system in figure 1a is given by 
the integral

$$
\begin{aligned}
\sigma_{i, j}= & \int_{-\infty}^{+\infty}\left[m f_{0}\left(\eta_{i}, \eta_{j}\right)\right. \\
& \left.+\frac{\kappa_{i, j}}{2}\left(\left(\frac{\mathrm{d} \eta_{i}}{\mathrm{dx}}\right)^{2}+\left(\frac{\mathrm{d} \eta_{j}}{\mathrm{dx}}\right)^{2}\right)\right] \mathrm{dx},
\end{aligned}
$$

with $\mathrm{x}$ the coordinate perpendicular to the grain boundary. For a grain boundary in local equilibrium, the profiles $\eta_{i}(\mathrm{x})$ and $\eta_{j}(\mathrm{x})$ adapt a shape that minimizes the functional (5) and satisfies the boundary conditions

$$
\begin{array}{rlrl}
\eta_{i}=1 \quad \text { and } & \eta_{j}=0 & \text { for } & \mathrm{x} \rightarrow-\infty \\
\eta_{i}=0 \quad \text { and } \eta_{j}=1 & \text { for } & \mathrm{x} \rightarrow+\infty \\
\frac{\mathrm{d} \eta_{i}}{\mathrm{dx}}=\frac{\mathrm{d} \eta_{j}}{\mathrm{dx}}=0 & \text { for } & \mathrm{x} \rightarrow \pm \infty .
\end{array}
$$

According to the principles of variational calculus ${ }^{32}$, the functions $\eta_{i}(\mathrm{x})$ and $\eta_{j}(\mathrm{x})$ that extremize the functional (5) satisfy

$$
\begin{aligned}
m \frac{\partial f_{0}}{\partial \eta_{i}}-\kappa_{i, j}\left(\frac{\mathrm{d}^{2} \eta_{i}}{\mathrm{dx}^{2}}\right) & =0 \\
m \frac{\partial f_{0}}{\partial \eta_{j}}-\kappa_{i, j}\left(\frac{\mathrm{d}^{2} \eta_{j}}{\mathrm{d \textrm {x } ^ { 2 }}}\right) & =0
\end{aligned}
$$

or, equivalently, the integrated equation

$$
m f_{0}-\frac{\kappa_{i, j}}{2}\left[\left(\frac{\mathrm{d} \eta_{i}}{\mathrm{~d} x}\right)^{2}+\left(\frac{\mathrm{d} \eta_{j}}{\mathrm{~d} x}\right)^{2}\right]=\mathrm{ct}=0,
$$

for $\gamma_{i, j}, \kappa_{i, j}$ and $m$ constants. Evaluation of the left hand side of equation (8) at $\pm \infty$ and considering the boundary conditions (6), gives that $\mathrm{ct}=0$. Rearrangement of equation (8) then yields

$$
\frac{\mathrm{d} \eta_{i}}{\mathrm{dx}}=-\sqrt{\frac{2 m f_{0}}{\kappa_{i, j}\left(1+\left(\frac{\mathrm{d} \eta_{j}}{\mathrm{~d} \eta_{i}}\right)^{2}\right)}}
$$

and

$$
\frac{\mathrm{d} \eta_{j}}{\mathrm{dx}}=\sqrt{\frac{2 m f_{0}}{\kappa_{i, j}\left(1+\left(\frac{\mathrm{d} \eta_{i}}{\mathrm{~d} \eta_{j}}\right)^{2}\right)}}
$$

were the boundary conditions (6) are taken into consideration. Combination of equations (5) and (8) gives

$$
\sigma_{i, j}=2 m \int_{-\infty}^{+\infty} f_{0}\left(\eta_{i}, \eta_{j}\right) \mathrm{dx}
$$


and, after changing the independent variable from $\mathrm{x}$ to $\eta_{i}$ using equation (9a),

$$
\begin{aligned}
\sigma_{i, j} & =2 m \int_{0}^{1} f_{0}\left(\eta_{i}, \eta_{j}\left(\eta_{i}\right)\right) \frac{\mathrm{dx}}{\mathrm{d} \eta_{i}} \mathrm{~d} \eta_{i} \\
& =\sqrt{2 m \kappa_{i, j}} \int_{0}^{1} \sqrt{f_{0}\left(\eta_{i}, \eta_{j}\left(\eta_{i}\right)\right)} \sqrt{1+\left(\frac{\mathrm{d} \eta_{j}\left(\eta_{i}\right)}{\mathrm{d} \eta_{i}}\right)^{2}} \mathrm{~d} \eta_{i} .
\end{aligned}
$$

It is clear from relation (11) that the grain boundary energy is proportional to the square root of $\kappa_{i, j}$ and $m$. The remaining integral, however, is in general too complicated to solve analytically. If equations (7) are combined and the partial derivatives of $f_{0}$ are worked out, it follows that

$$
\frac{\mathrm{d}\left(\frac{\mathrm{d} \eta_{j}}{\mathrm{dx}}\right)}{\mathrm{d}\left(\frac{\mathrm{d} \eta_{i}}{\mathrm{dx}}\right)}=\frac{\eta_{j}^{3}-\eta_{j}+2 \gamma_{i, j} \eta_{i}^{2} \eta_{j}}{\eta_{i}^{3}-\eta_{i}+2 \gamma_{i, j} \eta_{i} \eta_{j}^{2}}
$$

only depends on the model parameter $\gamma_{i, j}$ and, by consequence, $\mathrm{d} \eta_{j} / \mathrm{d} \eta_{i}$ and $\eta_{j}\left(\eta_{i}\right)$ do as well. The relation between the specific grain boundary energy and the model parameters can accordingly be written in the form

$$
\sigma_{i, j}=g\left(\gamma_{i, j}\right) \sqrt{\kappa_{i, j} m}
$$

Knowledge of the function $g\left(\gamma_{i, j}\right)$ and its inverse $g^{-1}\left(\gamma_{i, j}\right)$ would greatly facilitate the choice of the model parameters for a given grain boundary energy. Equations (7) for the phase field profiles are evaluated in section IID and integral (11) for the grain boundary energy in section II E. First, the effect of the parameter $\gamma_{i, j}$ on the landscape of the homogeneous free energy is examined.

\section{Homogeneous free energy density landscape}

For $\gamma_{i, j}>0.5$, the combinations of phase field variable values

$$
\left(\eta_{i}, \eta_{j}\right)=(0,0),( \pm 1,0),(0, \pm 1)
$$

and

$$
\left( \pm \frac{1}{\left.\sqrt{(} 2 \gamma_{i, j}+1\right)}, \pm \frac{1}{\left.\sqrt{(} 2 \gamma_{i, j}+1\right)}\right)
$$

satisfy the conditions

$$
\frac{\partial f_{0}}{\partial \eta_{i}}=\eta_{i}^{3}-\eta_{i}+2 \gamma_{i, j} \eta_{i} \eta_{j}^{2}=0
$$




$$
\frac{\partial f_{0}}{\partial \eta_{j}}=\eta_{j}^{3}-\eta_{j}+2 \gamma_{i, j} \eta_{i}^{2} \eta_{j}=0
$$

for the extremal and saddle points of $f_{0}\left(\eta_{i}, \eta_{j}\right)$. Only positive values of $\eta_{i}$ and $\eta_{j}$ will be considered in the further analysis. The point $(0,0)$, where $f_{0}=0.25$, is a local maximum and the points $(1,0),(0,1)$ are degenerate minima, where $f_{0}=0$. The point $\left(\frac{1}{\sqrt{\left(2 \gamma_{i, j}+1\right)}}, \frac{1}{\sqrt{\left(2 \gamma_{i, j}+1\right)}}\right)$ is a saddle point. Substitution of $\left(\eta_{i}, \eta_{j}\right)=\left(\eta_{\text {saddle }}, \eta_{\text {saddle }}\right)$, with

$$
\eta_{\text {saddle }}=\frac{1}{\sqrt{2 \gamma_{i, j}+1}}
$$

into (4), gives

$$
f_{0}\left(\eta_{\text {saddle }}\right)=\frac{2 \gamma_{i, j}-1}{4\left(2 \gamma_{i, j}+1\right)}
$$

Both, the position and height of the saddle point depend on the model parameter $\gamma_{i, j}$. Furthermore, it follows from the conditions (14) that for $\gamma=0.5$, there is an infinite number of minima, namely all points on the circle $\eta_{i}^{2}-1+\eta_{j}^{2}=0$, and for $\gamma_{i, j}<0.5$, the absolute minimum is at $\left(\frac{1}{\sqrt{\left(2 \gamma_{i, j}+1\right)}}, \frac{1}{\sqrt{\left(2 \gamma_{i, j}+1\right)}}\right)$ instead of $(0,1)$ and $(1,0)$. A free energy with $\gamma_{i, j} \leq 0.5$ is thus inappropriate for the description of multi-grain structures.

Figure 2 shows contour plots of the homogeneous free energy density (4) as a function of two phase field variables $\eta_{i}$ and $\eta_{j}$ and for different values of $\gamma_{i, j}$. For $\gamma_{i, j}>0.5$, there are clearly localized minima at $(1,0)$ and $(0,1)$. The saddle points are indicated with a cross. For all values of $\gamma_{i, j}$, the free energy is symmetrical with respect to the diagonal $\eta_{i}=\eta_{j}$. It is not symmetrical with respect to the diagonal $\eta_{i}=1-\eta_{j}$, since the contribution of the term $\gamma_{i, j} \eta_{i}^{2} \eta_{j}^{2}$ increases with the values of the phase field variables. Moreover, for larger values of $\gamma_{i, j}$, the contours deviate more towards the corner $\left(\eta_{i}, \eta_{j}\right)=(0,0)$ and the saddle point is at smaller values of the phase field variables. The relation $\eta_{j}\left(\eta_{i}\right)$ between the values of the phase field variables along their profiles across a grain boundary in local equilibrium is indicated using a bold line and will be commented in section II D.

The evolution of $f_{0}$ along the diagonal $\eta_{i}=\eta_{j}$ is plotted for different $\gamma_{i, j}$ in figure 3 . For all values of $\gamma_{i, j}$, there is a local maximum $f_{0}=0.25$ at $\eta_{i}=\eta_{j}=0$. There would be a minimum at $\eta_{i}=\eta_{j}=1$, for $\gamma_{i, j}=0$. However, for $\gamma_{i, j}>0.5$, the contribution $\gamma_{i, j} \eta_{i}^{2} \eta_{j}^{2}$, which increases as $\eta_{1}=\eta_{2}$ approaches 1 , prevents the minimum at $\eta_{i}=\eta_{j}=1$ and introduces a saddle point (minimum along the curve for $f_{0}\left(\eta_{i}, \eta_{j}\right)$ ). The function values increase and the saddle point shifts towards smaller values, for increasing values of $\gamma_{i, j}$. For $\gamma_{i, j}=1.5$, the saddle point is at $\eta_{i}=\eta_{j}=0.5$. 

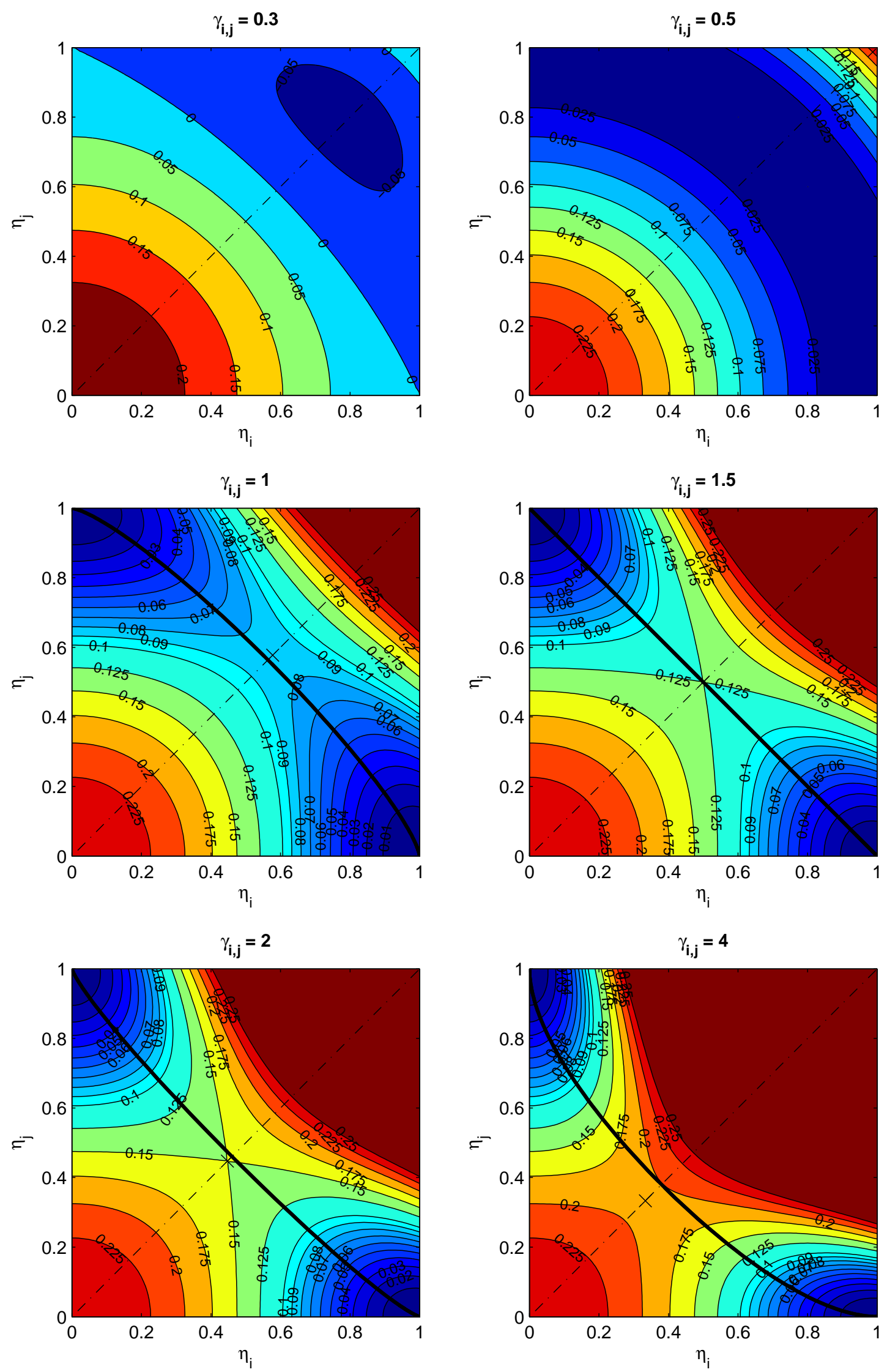

FIG. 2: Contour plots of $f_{0}\left(\eta_{i}, \eta_{j}\right)$, for different values of the model parameter $\gamma_{i, j}$. The position 10

of the saddle point is indicated with a cross. The dotted line along $\eta_{i}=\eta_{j}$ makes clear that the homogeneous free energy is symmetrical with respect to both order parameters. The bold black lines indicate the paths $\eta_{j}\left(\eta_{i}\right)$ across a grain boundary in local equilibrium. 


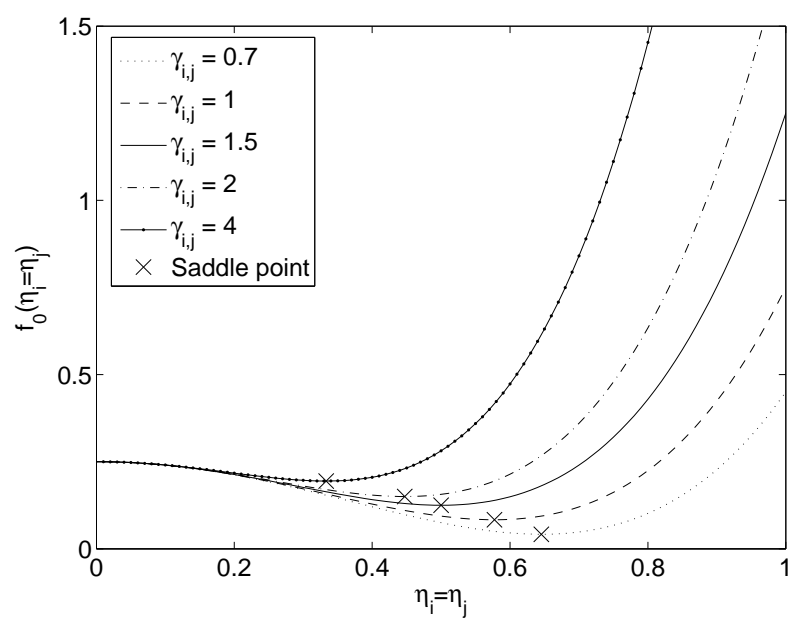

FIG. 3: Evolution of $f_{0}$ along the diagonal $\eta_{i}=\eta_{j}$ for different values of $\gamma_{i, j}$. The saddle point is indicated with a cross.

If more phase field variables are considered, the degenerate minima of the homogeneous free energy (4) are located at $\left(\eta_{i}=1, \eta_{j \neq i}=0\right)$ and there is a local maximum at $\eta_{i}=0$, assuming $\gamma>0.5$ and $i, j=1 \ldots p$. Moreover, there are saddle points at

$$
\left(\eta_{i}=\eta_{j}=\frac{1}{\sqrt{1+(2-1) \gamma}}, \eta_{k \neq i, j}=0\right)
$$

for all combinations of 2 phase field variables and

$$
\left(\eta_{i}=\eta_{j}=\eta_{k}=\frac{1}{\sqrt{1+(3-1) \gamma}}, \eta_{l \neq i, j, k}=0\right)
$$

for all combinations of 3 phase field variables, et cetera for higher order combinations. Because of the term $\gamma \sum_{i<j} \eta_{i}^{2} \eta_{j}^{2}$, each extra phase field variable with a value different from 0 , gives a positive contribution to the local free energy density. By consequence, within grains only 1 of the phase field variables differs from 0 (it equals 1), and at grain boundaries and multi-junctions, only those phase field variables representing the neighboring grains, are different from zero.

\section{Interfacial profiles}

The equilibrium profiles of $\eta_{i}$ and $\eta_{j}$ are given by the differential equations (7) in combination with the boundary conditions (6). In this section, the differential equations are 
first solved analytically for symmetrical phase field profiles $\left(\gamma_{i, j}=1.5\right)$. Then, numerically calculated profiles are analyzed for the general case.

\section{Analytical calculation of symmetrical profiles}

In the present study, a 'symmetrical profile' is a profile for which $\eta(\mathrm{x})=1-\eta(-\mathrm{x})$, assuming that the central plane of the diffuse grain boundary region is located at $\mathrm{x}=0$. Since the free energy formulation (3) - (4) is symmetrical with respect to $\eta_{i}$ and $\eta_{j}$, the following relations exist for symmetrical phase field profiles $\eta_{i}(\mathrm{x})$ and $\eta_{j}(\mathrm{x})$ across a grain boundary in local equilibrium:

$$
\begin{gathered}
\eta_{j}=1-\eta_{i}, \\
\frac{\mathrm{d} \eta_{j}}{\mathrm{dx}}=-\frac{\mathrm{d} \eta_{i}}{\mathrm{dx}}
\end{gathered}
$$

and

$$
\frac{\mathrm{d}^{2} \eta_{j}}{\mathrm{dx}^{2}}=-\frac{\mathrm{d}^{2} \eta_{i}}{\mathrm{dx}^{2}}
$$

Consequently, for symmetrical profiles the path $\eta_{i}\left(\eta_{j}\right)$ and its inverse are known and have a simple form, namely relation (17a). Furthermore, it follows from the conditions (17) that the profiles of the phase field variables cross at $\eta_{i}=\eta_{j}=\eta_{\text {interf }}=0.5$ and that $\mathrm{d} \eta_{i} / \mathrm{d} \eta_{j}=-1$ and $\mathrm{d}\left(\mathrm{d} \eta_{i} / \mathrm{dx}\right) / \mathrm{d}\left(\mathrm{d} \eta_{j} / \mathrm{dx}\right)=-1$ along the profiles. Application of condition (17c) to the differential equations (7), gives that the 2 phase field profiles across a diffuse interface can only be symmetrical if at every point $\mathrm{x}$ along the profile

$$
\frac{\partial f_{0}}{\partial \eta_{j}}=-\frac{\partial f_{0}}{\partial \eta_{i}}
$$

or, using equation (14) for the partial derivatives of $f_{0}$ with $\eta_{j}=1-\eta_{i}$,

$$
\begin{gathered}
-\left(1+2 \gamma_{i, j}\right) \eta_{i}^{3}+\left(3+2 \gamma_{i, j}\right) \eta_{i}^{2}-2 \eta_{i} \\
=-\left(1+2 \gamma_{i, j}\right) \eta_{i}^{3}+4 \gamma_{i, j} \eta_{i}^{2}-\left(2 \gamma_{i, j}-1\right) \eta_{i}
\end{gathered}
$$

This requirement can only be satisfied at every point along the interface for $\gamma_{i, j}=1,5$.

Since for symmetrical profiles $\eta_{j}\left(\eta_{i}\right)=1-\eta_{i}$, it is straightforward to eliminate 1 of the phase field variables from the equations (7)-(9) for a boundary in local equilibrium. The 
functional $f_{0}$ and its partial derivatives can be written as

$$
\begin{aligned}
f_{0}\left(\eta_{i}, 1-\eta_{i}\right)= & \left(\frac{\eta_{i}^{4}}{4}-\frac{\eta_{i}^{2}}{2}\right) \\
& +\left(\frac{\left(1-\eta_{i}\right)^{4}}{4}-\frac{\left(1-\eta_{i}\right)^{2}}{2}\right) \\
& +\gamma_{i, j} \eta_{i}^{2}\left(1-\eta_{i}\right)^{2}+\frac{1}{4} \\
= & \left(\frac{1}{2}+\gamma_{i, j}\right) \eta_{i}^{2}\left(1-\eta_{i}\right)^{2},
\end{aligned}
$$

and

$$
\begin{aligned}
\frac{\partial f_{0}}{\partial \eta_{i}}\left(\eta_{i}, 1-\eta_{i}\right)= & \left(1+2 \gamma_{i, j}\right) \eta_{i}^{3} \\
& -4 \gamma_{i, j} \eta_{i}^{2} \\
& +\left(2 \gamma_{i, j}-1\right) \eta_{i} .
\end{aligned}
$$

Analogous expressions are obtained for $\partial f_{0} / \partial \eta_{i}$ as a function of $\eta_{j}$ and $\partial f_{0} / \partial \eta_{j}$ as a function of $\eta_{i}$ or $\eta_{j}$. As a result, for symmetrical profiles, the differential equations (7) become

$$
\begin{aligned}
& \frac{\mathrm{d}^{2} \eta_{i}}{\mathrm{dx}^{2}}=\frac{m}{\kappa_{i, j}} 2 \eta_{i}\left(2 \eta_{i}^{2}-3 \eta_{i}+2\right) \\
& \frac{\mathrm{d}^{2} \eta_{j}}{\mathrm{dx}^{2}}=\frac{m}{\kappa_{i, j}} 2 \eta_{j}\left(2 \eta_{j}^{2}-3 \eta_{j}+2\right)
\end{aligned}
$$

and the integrated equations (9)

$$
\begin{aligned}
& \frac{\mathrm{d} \eta_{i}}{\mathrm{dx}}=-\sqrt{\frac{2 m}{\kappa_{i, j}}} \eta_{i}\left(1-\eta_{i}\right) \\
& \frac{\mathrm{d} \eta_{j}}{\mathrm{dx}}=\sqrt{\frac{2 m}{\kappa_{i, j}}} \eta_{j}\left(1-\eta_{j}\right),
\end{aligned}
$$

where it is applied that $\gamma_{i, j}=1.5$ and $\mathrm{d} \eta_{i} / \mathrm{d} \eta_{j}=\mathrm{d} \eta_{j} / \mathrm{d} \eta_{i}=-1$. It can be verified that the profiles

$$
\begin{aligned}
& \eta_{i}(\mathrm{x})=\frac{1}{2}\left[1-\tanh \left(\sqrt{\frac{m}{2 \kappa_{i, j}} \mathrm{x}}\right)\right] \\
& \eta_{j}(\mathrm{x})=\frac{1}{2}\left[1+\tanh \left(\sqrt{\frac{m}{2 \kappa_{i, j}} \mathrm{x}}\right)\right]
\end{aligned}
$$

satisfy (22) and (23) in combination with the boundary conditions (6). 


\section{Numerical calculation of arbitrary profiles}

It follows from requirement (19) that, in general, the phase field profiles are not symmetrical, except for $\gamma_{i, j}=1.5$. For asymmetrical profiles, the ratios $\left(\mathrm{d}^{2} \eta_{i} / \mathrm{dx}^{2}\right) /\left(\mathrm{d}^{2} \eta_{j} / \mathrm{dx}^{2}\right)$ and $\left(\mathrm{d} \eta_{i} / \mathrm{dx}\right) /\left(\mathrm{d} \eta_{j} / \mathrm{dx}\right)$ are not constant along the profiles and the relation between $\eta_{i}(\mathrm{x})$ and $\eta_{j}(\mathrm{x})$ is complex. It was not possible to identify analytical expressions for the profiles $\eta_{i}(\mathrm{x})$ and $\eta_{j}(\mathrm{x})$, which satisfy the differential equations (7). Therefore, numerical simulations were performed to study the effect of the model parameters $m, \kappa_{i, j}$ and $\gamma_{i, j}$ on the equilibrium shape of the profiles, using the 1-dimensional two-grain geometry shown in figure 1a. In the simulations, the phase field profiles evolved towards their equilibrium shape, starting from a sharp transition at the grain boundary. To obtain a well resolved representation of the profiles, an extremely fine grid was used: the grid spacing was 0.1 or smaller, so that there were at least 100 g.p. between the position $\mathrm{x}=-5$ and $\mathrm{x}=5$ (see figures 4 and 7 ). The distance between the markers on the curves in the figures is many times the grid spacing used in the numerical calculations.

Numerically calculated phase field profiles are shown for different values of $\gamma_{i, j}$ and a constant ratio $\kappa_{i, j} / m$ in figure $4 \mathrm{a}$ and for different ratios $\kappa_{i, j} / m$ and constant $\gamma_{i, j}$ in figure $4 \mathrm{~b}$. Comparison of the numerically calculated profiles for $\gamma_{i, j}=1.5$ with the analytical profiles (24), indicates that the numerically calculated profiles converge to the analytical solution of equations (7) and that the grid spacing results in well resolved phase field profiles. Since the homogeneous free energy is symmetrical with respect to the phase field variables, the profiles $\eta_{i}(\mathrm{x})$ and $\eta_{j}(\mathrm{x})$ are each others mirror image with respect to the center of the diffuse interface region, where the phase field variables intersect; this involves that $\eta_{i}(\mathrm{x})=\eta_{j}(-\mathrm{x})$. The value at which the phase field profiles intersect, $\eta_{\text {interf }}$, depends only on the parameter $\gamma_{i, j}$. This also follows from equations (9), since $f_{0}$ and $\mathrm{d} \eta_{i} / \mathrm{d} \eta_{j}$ are only a function of the model parameter $\gamma_{i, j}$, and the parameters $\kappa_{i, j}$ and $m$ are constant along the interfacial profiles. Except for $\gamma_{i, j}=1.5$, the profiles of the phase field variables themselves are not symmetrical and their local sum is not constant along the profiles. Condition (17a) is accordingly not satisfied. For fixed $\gamma_{i, j}$-values, the width of the interfacial profiles is proportional with $\sqrt{\kappa_{i, j} / m}$ (see also section II F).

In figure $4 \mathrm{c}$, the gradients of the phase field profiles are plotted as a function of the distance from the middle of the interface for different $\gamma_{i, j}$-values and a constant $m / \kappa$-ratio. 

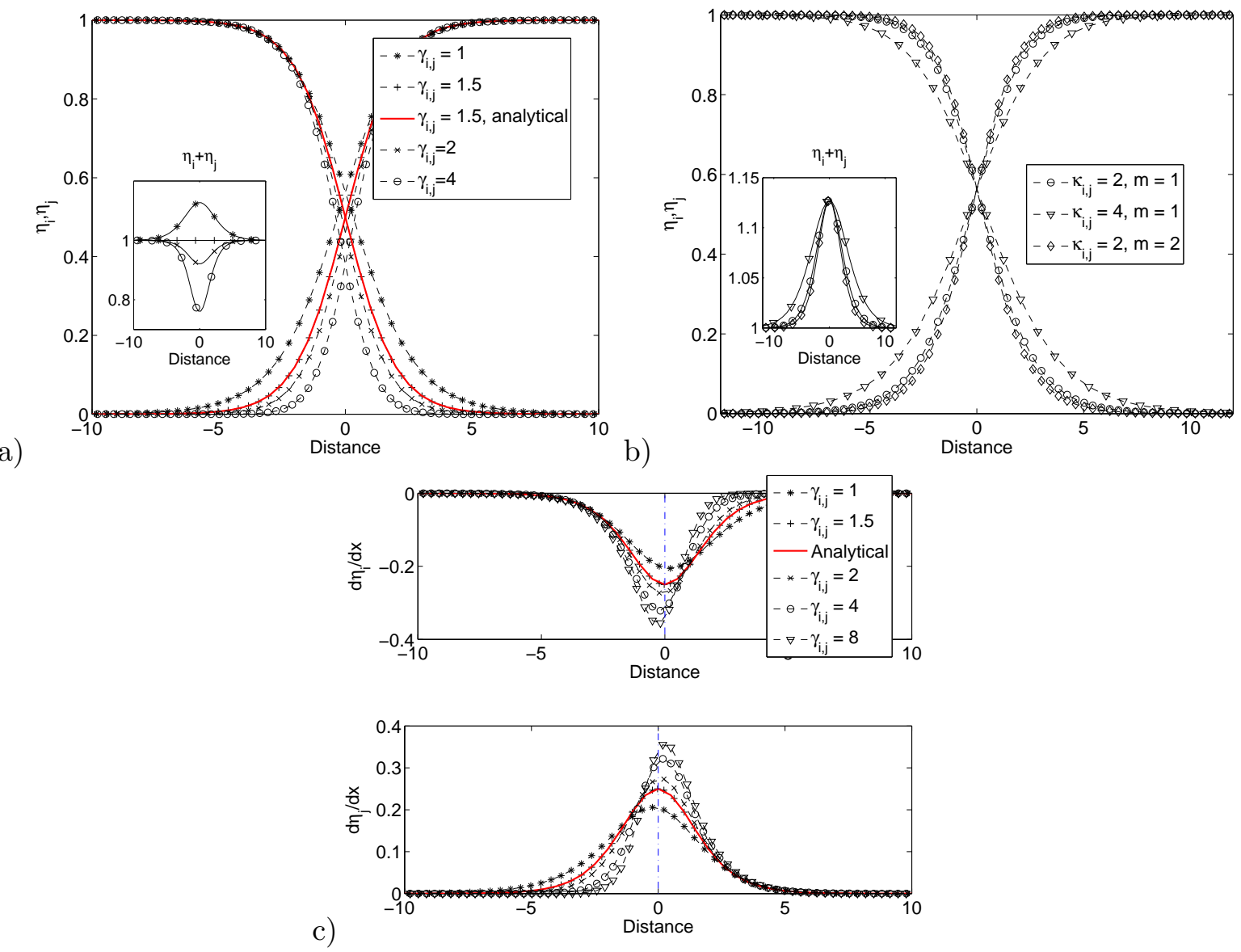

FIG. 4: a) Numerically calculated equilibrium profiles for the phase field variables $\eta_{i}(\mathrm{x})$ and $\eta_{j}(\mathrm{x})$ across a grain boundary, for $\kappa_{i, j}=2, m=1$ and different values for $\gamma_{i, j}$. b) Numerically calculated equilibrium profiles for the phase field variables $\eta_{i}(\mathrm{x})$ and $\eta_{j}(\mathrm{x})$ across a grain boundary, for $\gamma_{i, j}=1$ and different combinations of the parameters $\kappa_{i, j}$ and $m$. c) Evolution of $\mathrm{d} \eta_{i} / \mathrm{dx}$ and $\mathrm{d} \eta_{j} / \mathrm{dx}$ along the interfacial profile for $\kappa_{i, j}=2, m=1$ and different values for $\gamma_{i, j}$. In both figure, the analytical curves for $\gamma_{i, j}=1.5$ are added.

For $\gamma_{i, j}=1.5$, the curves are symmetrical with respect to $\mathrm{x}=0$ and the local gradients of $\eta_{i}$ and $\eta_{j}$ are equal in magnitude. By consequence, $\mathrm{d} \eta_{i} / \mathrm{d} \eta_{j}=\mathrm{d} \eta_{j} / \mathrm{d} \eta_{i}=-1$ along the profiles. For $\gamma_{i, j}$ farther from 1.5, the curves become more asymmetric and the local gradients of $\eta_{i}$ and $\eta_{j}$ differ. At the middle of the interface, however, the gradients of the two profiles are maximal in absolute value and equal in size for all values of $\gamma_{i, j}$, giving $\left(\mathrm{d} \eta_{i} / \mathrm{d} \eta_{j}\right)_{\text {interf }}=\left(\mathrm{d} \eta_{j} / \mathrm{d} \eta_{i}\right)_{\text {interf }}=-1$. The graphs also show that for a given ratio $m / \kappa_{i, j}$, the maximal gradient at the middle of the interface increases with the value of $\gamma_{i, j}$. For fixed $\gamma_{i, j}$-values, the gradients along the phase field profiles are proportional with $\sqrt{m / \kappa_{i, j}}$ (see 
relations (9)).

Although the evolution of each phase field variable towards equilibrium is independent, there exists a relation between the local values of the two phase field variables, which are changing values across a diffuse boundary in local equilibrium. This relation $\eta_{j}\left(\eta_{i}(\mathrm{x})\right.$ ) (or $\left.\eta_{i}\left(\eta_{j}(\mathrm{x})\right)\right)$ depends on the value of the model parameter $\gamma_{i, j}$ and is plotted for a number of $\gamma_{i, j}$-values in figure $5 \mathrm{a}$. The paths of $\left(\eta_{i}, \eta_{j}\right)$-values across a diffuse grain boundary are also indicated on the free energy landscapes in figure 2. For $\gamma_{i, j}=1.5$, the path corresponds with the diagonal $\eta_{j}=1-\eta_{i}$ and goes through the saddle point of the homogeneous free energy at $\eta_{i}=\eta_{j}=0.5$. For $\gamma_{i, j}<>1.5$, the curves deviate towards the saddle point, however do not reach it. Figure $5 \mathrm{~b}$ shows how $\mathrm{d} \eta_{i} / \mathrm{d} \eta_{j}$ varies with the value of one of the phase field variables $\eta_{i}$. Conform with the results presented in figure $4 \mathrm{~b}, \mathrm{~d} \eta_{i} / \mathrm{d} \eta_{j}$ may vary between 0 and $-\infty$, but equals -1 at $\mathrm{x}=0$ for all values of $\gamma_{i, j}$. For symmetrical profiles, $\mathrm{d} \eta_{i} / \mathrm{d} \eta_{j}$ is constant and equal to -1 along the whole profile. .

In figure $6 \mathrm{a}$, the value at which the phase field variables cross, $\eta_{\text {interf }}$, is compared with the coordinates of the saddle point of the homogeneous free energy $\eta_{\text {saddle }}$, for a large range of $\gamma_{i, j}$-values. The difference between $\eta_{\text {interf }}$ and $\eta_{\text {saddle }}$ is relatively small for $\gamma_{i, j}$ between 0.5 and 2 and increases drastically for larger values of $\gamma_{i, j}$. For $\gamma_{i, j}=1.5, \eta_{\text {interf }}$ equals $\eta_{\text {saddle }}$, and for $\gamma_{i, j} \rightarrow 0.5, \eta_{\text {interf }} \rightarrow \eta_{\text {saddle }}=1 / \sqrt{2}$, which is calculated from equation (15) with $\gamma_{i, j}=0.5$

\section{E. Grain boundary energy}

In diffuse interface models, the excess energy associated with a grain boundary is smeared out over the diffuse grain boundary region. Integration of the excess energy along the phase field profiles then gives the specific grain boundary energy, which is a measurable quantity.

\section{Spatial distribution of the excess energy}

The integrant in equation (5) gives the local excess energy density due to the presence of a grain boundary, which consists of a homogeneous contribution, $m f_{0}$, and a gradient contribution, $0.5 \kappa_{i, j}\left[\left(\mathrm{~d} \eta_{i} / \mathrm{dx}\right)^{2}+\left(\mathrm{d} \eta_{j} / \mathrm{dx}\right)^{2}\right]$. It follows from relation (8) that both contributions are equal at every position along the profiles. 


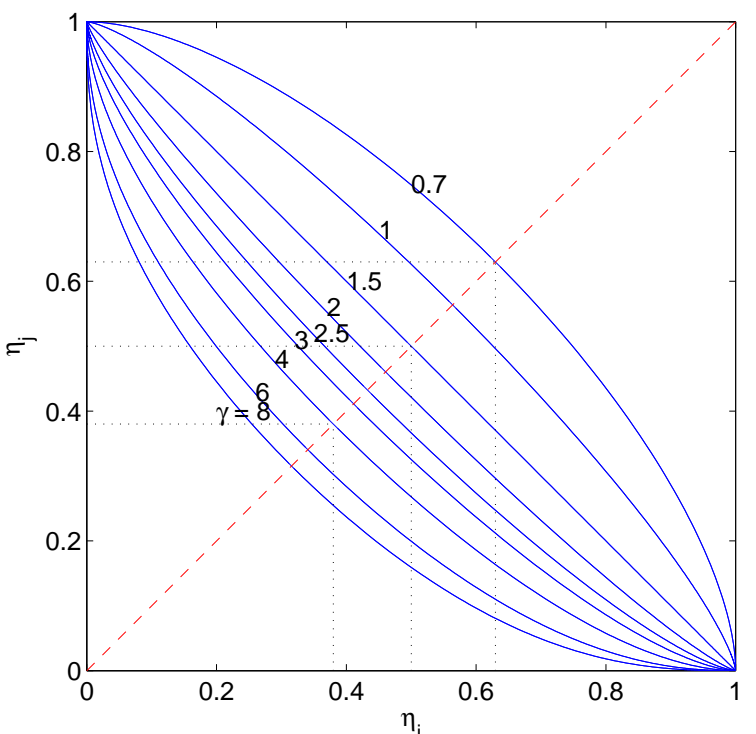

a)

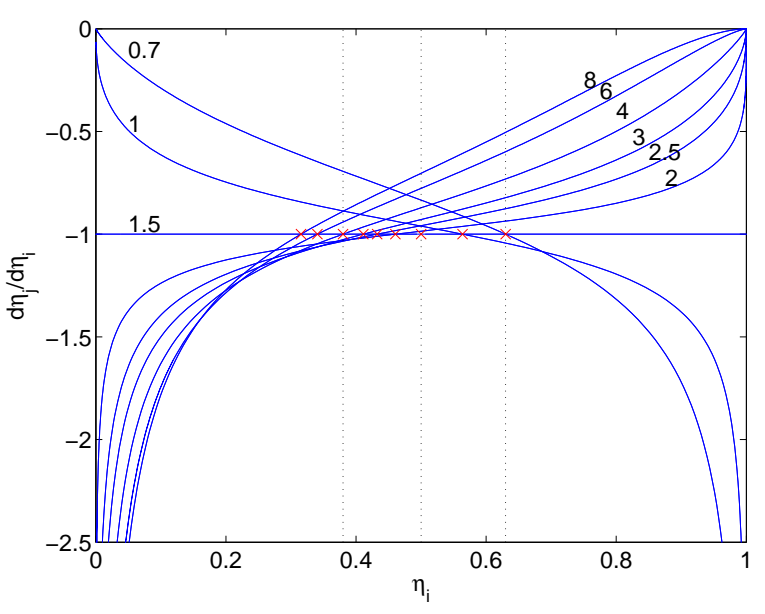

FIG. 5: Variation of a) $\eta_{j}$ and b) $\mathrm{d} \eta_{j} / \mathrm{d} \eta_{i}$ as a function of $\eta_{i}$ along equilibrium phase field profiles across a grain boundary, obtained from numerical simulations for different values of the parameter $\gamma_{i, j}$ (curves for $\mathrm{d} \eta_{i} / \mathrm{d} \eta_{j}$ as a function of $\eta_{j}$ are identical). The intersections of the broken line (red) for $\eta_{i}=\eta_{j}$ with the $\eta_{j}\left(\eta_{i}\right)$-curves give the values at which the phase field profiles cross at the center of the diffuse grain boundary region. For the different values of $\gamma_{i, j}, \eta_{\text {interf }}$ is indicated with a (red) cross.

For $\gamma_{i, j}=1.5$, the energy density curves can be calculated analytically from equation (20) and using expressions (24), giving

$$
\begin{gathered}
m f_{0}\left(\eta_{i}(\mathrm{x}), \eta_{j}(\mathrm{x})\right)=2 m \eta_{i}^{2}\left(1-\eta_{i}\right)^{2} \\
=\frac{m}{8}\left(\cosh \left(\sqrt{\frac{m}{2 \kappa_{i, j}} \mathrm{x}}\right)\right)^{-4}
\end{gathered}
$$



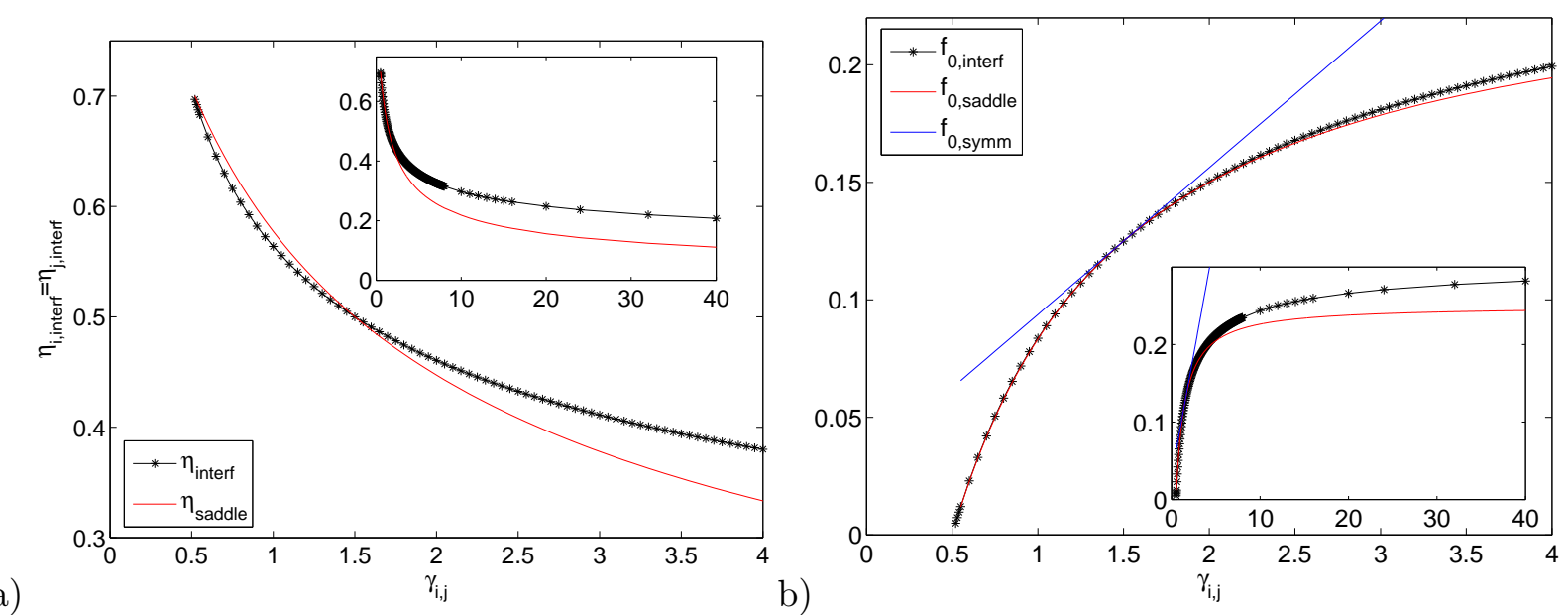

FIG. 6: $\eta_{\text {interf }}$ as a function of $\gamma_{i, j}$, obtained from numerical simulations, compared with the

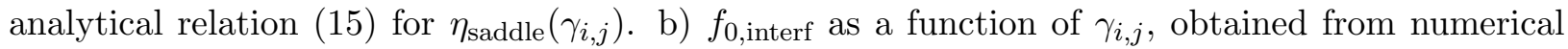
simulations, compared with relations (16) for $f_{0, \text { saddle }}\left(\gamma_{i, j}\right)$ and $(20)$ for $f_{0, \text { symm }}\left(\gamma_{i, j}\right)$, evaluated for different values of $\gamma_{i, j}$. The latter assumes that the profiles are symmetrical and intersect at $\eta_{i}=\eta_{j}=0.5$.

for the homogeneous energy density, and

$$
\begin{aligned}
\frac{\kappa_{i, j}}{2}\left[\left(\frac{\mathrm{d} \eta_{i}}{\mathrm{dx}}\right)^{2}\right. & \left.+\left(\frac{\mathrm{d} \eta_{j}}{\mathrm{dx}}\right)^{2}\right]=\kappa_{i, j}\left(\frac{\mathrm{d} \eta_{i}}{\mathrm{dx}}\right)^{2} \\
& =\frac{m}{8}\left(\cosh \left(\sqrt{\frac{m}{2 \kappa_{i, j}} \mathrm{x}}\right)\right)^{-4}
\end{aligned}
$$

for the gradient energy density. The spatial distribution of the excess energy density due to the presence of a grain boundary is given by the sum of both terms. The curves reach a maximum at the middle of the interface, where $f_{0, \text { interf }}=m / 8$.

For arbitrary profiles $\left(\gamma_{i, j}<>1.5\right)$, the free energy curves and their maximum at the middle of the diffuse grain boundary region were calculated numerically for the equilibrium phase field profiles described in the previous section. The spatial variation of the homogeneous and gradient contributions in the energy density are plotted in figures 7a-b for different values of the model parameters $\gamma_{i, j}, \kappa_{i, j}$ and $m$. For all parameter values, the curves are symmetrical and have a maximum at the middle of the profile. The major contribution to the grain boundary energy is concentrated near the center of the diffuse grain boundary region. Comparison of figures $7 \mathrm{a}-\mathrm{b}$ with figures $4 \mathrm{a}-\mathrm{b}$, reveals that the tails of the energy density peak are much shorter than those of the profiles of the phase field variables. For 

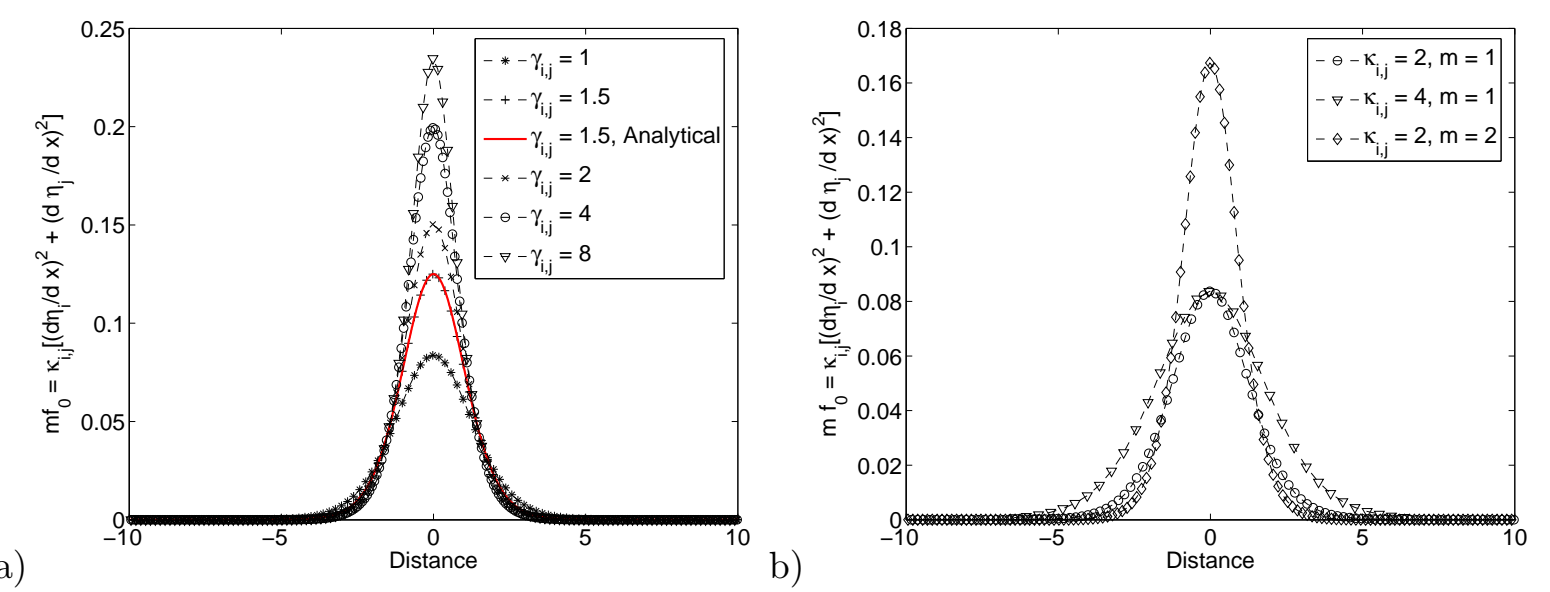

a)

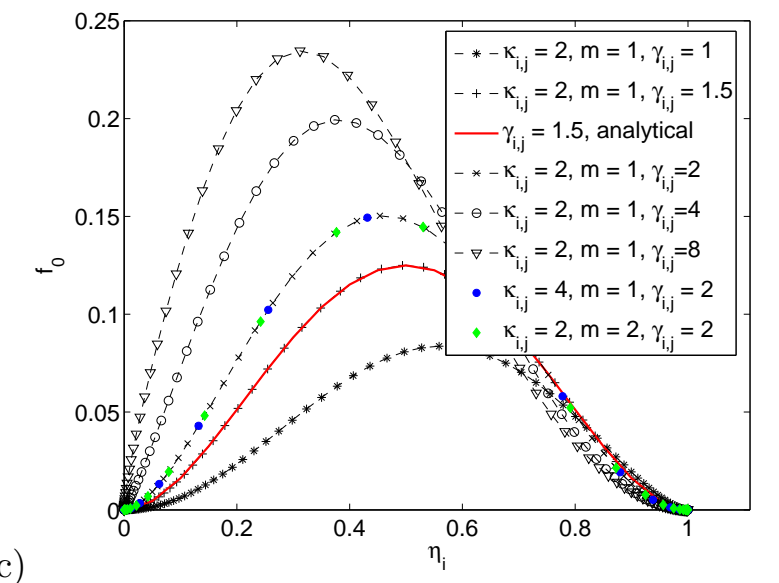

FIG. 7: a) Profiles of the homogeneous and gradient energy density variations across a grain boundary for $\kappa_{i, j}=2, m=1$ and different values of $\gamma_{i, j}$. For $\gamma_{i, j}=1.5$, the analytical relations are added. b) Profiles of the energy density variations across a grain boundary for $\gamma_{i, j}=1$ and different combinations of the values of $\kappa_{i, j}=4, m=1$. c) Evolution of $f_{0}$ along numerically calculated paths for $\eta_{j}\left(\eta_{i}\right)$. The curves were obtained for $\kappa_{i, j}=2, m=1$ and different values of $\gamma_{i, j}$. A number of points, obtained for $\kappa_{i, j}=4, m=1$ and $\kappa_{i, j}=2, m=2$ with $\gamma_{i, j}=2$ are added.

constant $\kappa_{i, j}$ and $m$, the maximum of the curves increases with increasing $\gamma_{i, j}$. For constant $\gamma_{i, j}$, the hight of the peak is proportional with $m$, whereas $\kappa_{i, j}$ has no effect on the height. Since the phase field profiles are wider for larger values of the ratio $\kappa / m$, a larger value for $\kappa / m$ accordingly results in a wider energy density peak in figure $7 \mathrm{~b}$.

The evolution of $f_{0}$ across a diffuse grain boundary as a function of one of the phase field variables is plotted for different values of $\gamma_{i, j}$ in figure $7 \mathrm{c}$. The maximum of the curve is always located at $\eta_{i}=\eta_{\text {interf }}$. The extra points for various values of $\kappa_{i, j}$ and $m$ on the curve for $\gamma_{i, j}=2$ show that the paths $\left(\eta_{i}, \eta_{j}\left(\eta_{i}\right)\right)$ and $\left(\eta_{i}, f_{0}\left(\eta_{i}, \eta_{j}\left(\eta_{i}\right)\right)\right)$ across a diffuse grain 
boundary depend only on the model parameter $\gamma_{i, j}$.

The gradients of the phase field profiles are proportional to the square root of the local value of the homogeneous energy density (see equation 9). By consequence, the maximum of $f_{0}$ along the profiles determines the stability and accuracy conditions for the numerical solution of the phase field equations. The value of $f_{0}$ at the middle of the diffuse interface region, $f_{0, \text { interf }}=f_{0}\left(\eta_{\text {interf }}, \eta_{\text {interf }}\right)$ was obtained for a large range of $\gamma_{i, j}$-values from the numerically calculated profiles. The data points are plotted in figure $6 \mathrm{~b}$ and compared with the analytical relations (16) for $f_{0, \text { saddle }}\left(\gamma_{i, j}\right)$ and (20) for $f_{0, \text { symm }}\left(\gamma_{i, j}\right)$. As was shown in figures 2 and $6 \mathrm{a}$, the path $\eta_{j}\left(\eta_{i}\right)$ across an interface in equilibrium does not reach the saddle point of $f_{0}$, except for $\gamma_{i, j}=1.5$. By consequence, the numerically determined values for

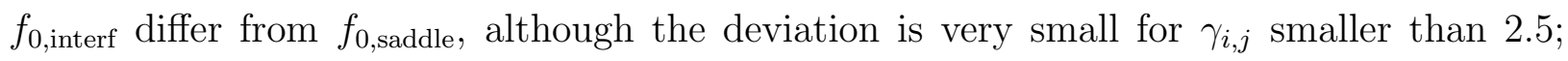
see figure 8 for a quantitative evaluation of the relative deviation. For larger values of $\gamma_{i, j}$, the curves for $f_{0 \text {,interf }}$ and $f_{0 \text {,saddle }}$ diverge. The line for $f_{0 \text {,symm }}$ was obtained by evaluating equation (20) for symmetrical interfaces, at $\eta_{i}=0.5$ and as a function of $\gamma_{i, j}$. It gives the maximum of $f_{0}$ along the diagonal $\eta_{j}=1-\eta_{i}$ for different values of $\gamma_{i, j}$. This curve reveals that the assumption of a symmetrical interface with $\eta_{j}=1-\eta_{i}$, and accordingly $\eta_{\text {interf }}=0.5$, cannot give a good approximation for $f_{0 \text {,interf }}$, the diffuse grain boundary width or the specific grain boundary energy.

\section{Specific grain boundary energy}

For symmetrical profiles, substitution of (17b) and (20) into (11), gives

$$
\begin{aligned}
\sigma_{i, j} & =2 \sqrt{m \kappa_{i, j}\left(\frac{1}{2}+\gamma_{i, j}\right)} \int_{0}^{1} \eta_{i}\left(1-\eta_{i}\right) \mathrm{d} \eta_{i} \\
& =\frac{1}{3} \sqrt{m \kappa_{i, j}\left(\frac{1}{2}+\gamma_{i, j}\right)} \\
& =\frac{\sqrt{2}}{3} \sqrt{m \kappa_{i, j}}
\end{aligned}
$$

for the specific grain boundary energy. The function $g\left(\gamma_{i, j}\right)$, introduced in equation (13), accordingly equals $\sqrt{2} / 3$ for $\gamma_{i, j}=1.5$. Furthermore, since for symmetrical interfaces $f_{0, \text { interf }}=f_{0, \text { saddle }}=1 / 8, g\left(\gamma_{i, j}\right)=\frac{4}{3} \sqrt{f_{0, \text { interf }}\left(\gamma_{i, j}\right)}=\frac{4}{3} \sqrt{f_{0, \text { saddle }}\left(\gamma_{i, j}\right)}$.

To characterize the function $g\left(\gamma_{i, j}\right)=\sigma_{i, j} / \sqrt{\kappa_{i, j} m}$, the integral expression of the specific grain boundary energy (5) was numerically evaluated for a large number of $\gamma_{i, j}$-values 


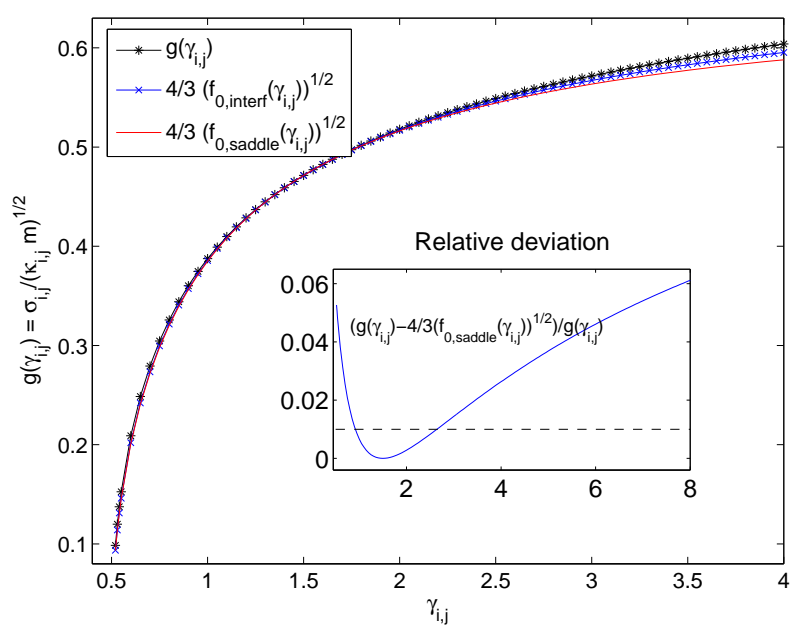

FIG. 8: Numerically calculated function values of $g\left(\gamma_{i, j}\right)=\sigma_{i, j} / \sqrt{\kappa_{i, j} m}$ and numerically calculated values of $4 / 3 \sqrt{\left(f_{0, \text { interf }}\left(\gamma_{i, j}\right)\right)}$ for a large number of $\gamma_{i, j}$-values, together with the analytical relation (16) for $4 / 3 \sqrt{\left(f_{0, \text { saddle }}\left(\gamma_{i, j}\right)\right)}$. The relative deviation between $g\left(\gamma_{i, j}\right)$ and $4 / 3 \sqrt{\left(f_{0 \text {,saddle }}\left(\gamma_{i, j}\right)\right)}$ is also shown as a function of $\gamma_{i, j}$.

between 0.52 and 40, using an interspacing $\Delta \gamma_{i, j} \leq 0.05$. Calculated function values are plotted as a function of $\gamma_{i, j}$ in figure 8 and compared with numerically calculated values for $4 / 3 \sqrt{f_{0, \text { interf }}\left(\gamma_{i, j}\right)}$ and with the analytical curve $4 / 3 \sqrt{f_{0, \text { saddle }}\left(\gamma_{i, j}\right)}$ obtained from equation (16). The figure shows that the three relations coincide for $\gamma_{i, j}=1.5$ and that for nearly symmetrical interfaces, the analytical equation $4 / 3 \sqrt{f_{0, \text { saddle }}\left(\gamma_{i, j}\right)}$ is a very accurate approximation for the functions $g\left(\gamma_{i, j}\right)$ and $4 / 3 \sqrt{f_{0, \text { interf }}\left(\gamma_{i, j}\right)}$. For $\gamma_{i, j}$ within the range [0.9 2.65], the relative error is smaller than $1 \%$, which is much smaller than the scatter on experimental data for grain boundary energies and mobilities, or the usual dicretization errors on the numerical solution of the phase field equations; the relative error is smaller than $2 \%$ for $\gamma_{i, j}$ in the range [0.75 3.45] and smaller than $5 \%$ for $\gamma_{i, j}$ in the range [0.53 6.5].

\section{F. Diffuse grain boundary width}

In phase field simulations for grain growth, the width of the diffuse grain boundaries is most often chosen based on computational considerations. To increase the length and time scale of the simulations, it is taken larger than the physical grain boundary thickness, however still several orders smaller than the mean grain size. Since, strictly spoken, the diffuse interface reaches to infinity, there are many possibilities to define a measure for the 
grain boundary width. For the further analysis, we propose a definition that is based on the absolute value of the gradients of the phase field profiles at $\mathrm{x}=0$ :

$$
\begin{aligned}
\ell_{g b} & =\frac{1}{\left|\left(\mathrm{~d} \eta_{i} / \mathrm{dx}\right)_{\mathrm{x}=0}\right|}=\frac{1}{\left|\left(\mathrm{~d} \eta_{j} / \mathrm{dx}\right)_{\mathrm{x}=0}\right|} \\
& =\sqrt{\frac{\kappa_{i, j}}{m f_{0, \text { interf }}}},
\end{aligned}
$$

which is obtained from equations (9) evaluated at $\mathrm{x}=0$ where $\mathrm{d} \eta_{i} / \mathrm{d} \eta_{j}=-1$ and $f_{0}=$ $f_{0, \text { interf }}$. Since the profiles are steepest at the middle of the diffuse grain boundary region, a grain boundary width defined in this way can be used in numerical criteria for stability and accuracy.

Evaluation of expression (29) for symmetrical interfaces using equation (23) with $f_{0}(1 / 2,1 / 2)=1 / 8$ or the derivatives $(24)$, gives

$$
\ell_{g b}=\sqrt{\frac{8 \kappa_{i, j}}{m}} .
$$

For asymmetrical profiles, the value of $f_{0 \text {,interf }}$ must be calculated numerically as a function of $\gamma_{i, j}$. As shown in figure $6 \mathrm{~b}, f_{0, \text { interf }}\left(\gamma_{i, j}\right)$ is approximated very well by the analytical relation (16), for $\gamma_{i, j}$ not too far from 1.5.

In figure 9, phase field profiles are shown for grain boundaries with different grain boundary energies, but equal width according to definition (29). All profiles have the same gradient at the center of the diffuse grain boundary region. Dotted lines are added as a graphical illustration of the definition for $\ell_{g b}$. The lines pass through the point $(0,0.5)$ and are parallel with the tangent to the phase field profiles at $\mathrm{x}=0$. Then, the grain boundary width equals the distance between the two intersections of these lines with the $\mathrm{x}$-axis.

\section{G. Grain boundary velocity}

Mostly $^{24,33-35}$, the velocity of curved interfaces in phase field models is derived assuming sharp interface conditions, where it is assumed that the width $\ell_{g b}$ of the grain boundary is much smaller than its radii of curvature $R_{1}$ and $R_{2}$. Consider thereto a grain with orientation $i$ embedded in another grain with orientation $j$, as illustrated in figure 1b. Furthermore, for every point on the grain boundary surface a curvilinear coordinate system $\left(\mathbf{r}, \mathbf{t}_{1}, \mathbf{t}_{2}\right)$ is defined with $\mathbf{r}$ pointing outwards in the direction of the normal to the surface and $\mathbf{t}_{1}$ and $\mathbf{t}_{2}$ 


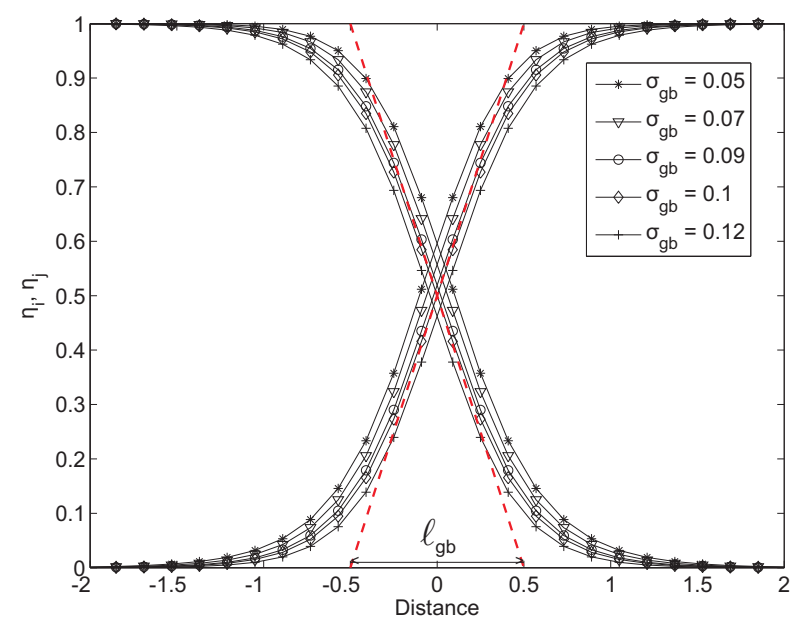

FIG. 9: Profiles of the phase field variables for grain boundaries with different grain boundary energies and equal diffuse grain boundary width, according to definition (29). The following parameter values, $m=0.6, \gamma=0.8262,1.0336,1.3156,1.4976$ and 1.9838 , and $\kappa=0.0371,0.0523$, $0.0675,0.0750$ and 0.0898 , were used, in order to obtain a grain boundary width $\ell_{g b}=1$ and grain boundary energies $\sigma_{g b}=0.05,0.07,0.09,0.1$, and 0.12 , The dotted lines give a graphical interpretation of definition (29).

tangential to the surface. Reformulation of the evolution equations (1) for the curvilinear coordinate system, gives ${ }^{24,33}$

$$
\begin{aligned}
\frac{\partial \eta_{i}}{\partial t}= & -L_{i, j}\left[m \frac{\partial f_{0}}{\partial \eta_{i}}\right. \\
& \left.-\kappa_{i, j}\left(\frac{\partial^{2} \eta_{i}}{\partial r^{2}}+\left(\frac{1}{R_{1}}+\frac{1}{R_{2}}\right) \frac{\partial \eta_{i}}{\partial r}\right)\right] \\
\frac{\partial \eta_{j}}{\partial t}= & -L_{i, j}\left[m \frac{\partial f_{0}}{\partial \eta_{j}}\right. \\
& \left.-\kappa_{i, j}\left(\frac{\partial^{2} \eta_{j}}{\partial r^{2}}+\left(\frac{1}{R_{1}}+\frac{1}{R_{2}}\right) \frac{\partial \eta_{j}}{\partial r}\right)\right],
\end{aligned}
$$

where it is applied that $\nabla \eta=(\partial \eta / \partial r) \mathbf{r}, \nabla^{2} \eta=\nabla \cdot \nabla \eta=\left(\partial^{2} \eta / \partial r^{2}\right)+(\nabla \cdot \mathbf{r})(\partial \eta / \partial r)$ and $(\nabla \cdot \mathbf{r})=1 / R_{1}+1 / R_{2}$ with $R_{1}$ and $R_{2}$ the principle radii of curvature.

For the considered boundary conditions

$$
\begin{aligned}
& \eta_{i}=1 \quad \text { and } \quad \eta_{j}=0 \quad \text { for } \quad r \rightarrow 0 \\
& \eta_{i}=0 \quad \text { and } \quad \eta_{j}=1 \quad \text { for } \quad r \rightarrow+\infty \\
& \frac{\mathrm{d} \eta_{i}}{\mathrm{~d} r}=\frac{\mathrm{d} \eta_{j}}{\mathrm{~d} r}=0 \quad \text { for } \quad r \rightarrow 0,+\infty,
\end{aligned}
$$


the term $\kappa_{i, j}\left(1 / R_{1}+1 / R_{2}\right) \partial \eta_{i} / \partial r$ forces the grain boundary to move towards its center of curvature $^{33}$. It was however derived ${ }^{34-36}$ that for $\ell_{g b} \ll R_{1}, R_{2}$, the effect of this extra term on the shape of the phase field profiles is negligible, and that conditions (7) are satisfied to leading order with $\mathrm{x}$ measured along the normal to the grain boundary. Moreover, if $\ell_{g b} \ll R_{1}, R_{2}$, the mean curvatures $1 / R_{1}$ and $1 / R_{2}$ can be considered to be constant within the thin grain boundary region where the phase field variables change their values. Under this assumption, all contours of equal $\eta_{i^{-}}$or $\eta_{j^{-}}$value move with the same velocity, the grain boundary velocity $v_{i, j}{ }^{33}$,

$$
\begin{aligned}
v_{i, j} & =\left(\frac{\partial r}{\partial t}\right)_{\eta_{i}, \eta_{j}=\mathrm{cte}}=\frac{\left(\frac{\partial \eta_{i / j}}{\partial t}\right)_{r}}{\left(\frac{\partial \eta_{i / j}}{\partial r}\right)_{t}} \\
& =-L_{i, j} \kappa_{i, j}\left(\frac{1}{R_{1}}+\frac{1}{R_{2}}\right),
\end{aligned}
$$

which is normal to the interface and negative when $\mathbf{r}$ is defined to point outwards as in figure 1b. In appendix A, equations (7) and (34) are derived in a more general way using an asymptotic analysis.

In the sharp interface limit, the velocity of the grain boundaries in phase field simulations is thus proportional with their local mean curvature. This is in analogy with the sharp interface Gibbs-Thomson law for grain boundary movement

$$
v_{g b}=-\mu_{g b} \sigma_{g b}\left(\frac{1}{R_{1}}+\frac{1}{R_{2}}\right),
$$

which gives the grain boundary velocity in terms of the grain boundary energy $\sigma_{g b}$ and mobility $\mu_{g b}$. Relation (35) is expressed in the same coordinate system as (34). Comparison of both equations yields a relation between the phase field model parameters and the grain boundary energy and mobility:

$$
\mu_{g b} \sigma_{g b}=\kappa_{i, j} L_{i, j}
$$

Relations (34) and (36) are valid for symmetrical and asymmetrical interfacial profiles. Equation (34) also shows that the velocity of a grain boundary in phase field simulations is not affected by the exact form of the homogeneous free energy, as long as it has localized minima with equal depth for all grain orientations.

To evaluate approximation (34), the shrinkage rate of a circular grain was determined from phase field simulations using varying grain boundary widths $\ell_{g b}$ and grid spacings 


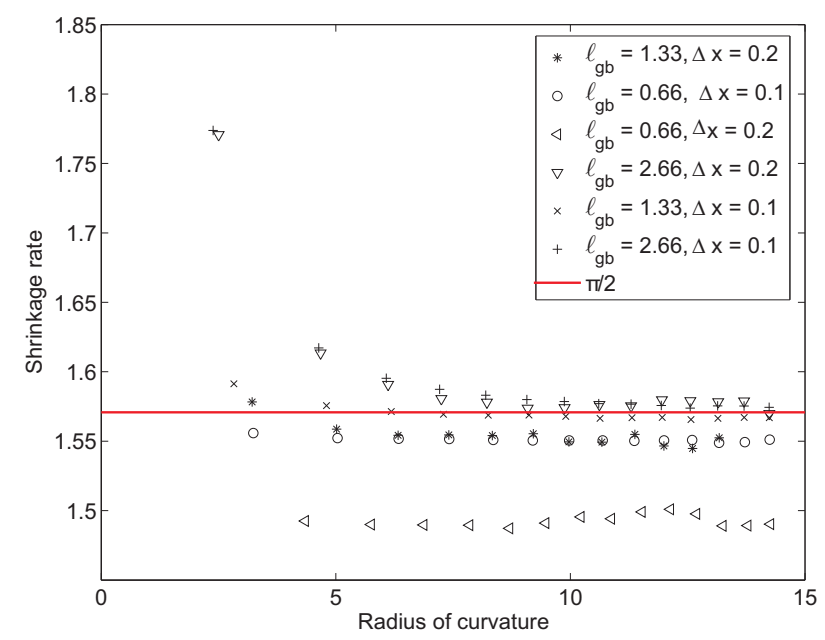

FIG. 10: Reduction in grain area per unit of time for a circular grain as a function of the grain radius, obtained from numerical simulations for $\sigma_{g b}=0.25$ and $\mu_{g b}=1$, and different grain boundary widths $\ell_{g b}$ and grid spacings $\Delta x$. For all simulations, $\gamma=1$. The values for $\kappa, m$ and $L$ were determined using relations (37) with the appropriate values for $\sigma_{g b}=0.25, \mu_{g b}=1$ and $\ell_{g b}$. The sharp interface approximation (34) predicts a shrinkage rate equal to $2 \pi \mu_{g b} \sigma_{g b}=\pi / 2$.

$\Delta x$. A standard finite difference discretization with explicit time stepping was used for the numerical solution. The results are plotted as a function of the grain radius in figure 10. The shrinkage rate is constant (which means that the velocity is proportional with the curvature) and the simulation results converge to the sharp interface approximation (34) for $\ell_{g b} / R<1 / 4$. A poor resolution results in a shrinkage rate too low: for the applied discretization technique, the relative deviations are approximately $0.25 \%, 1.3 \%$ and $5 \%$ for $\ell_{g b} / \Delta x$ equal to $13,6.6$ and 3.3 , for $R \gg \ell_{g b}$. These results confirm previous numerical tests $^{24}$.

\section{H. Calculation of the model parameters for uniform systems}

From the analysis in the previous subsections, relations can be derived that allow us to calculate appropriate parameters for the phase field model (1)-(3). The set of model parameters should reproduce a given grain boundary energy $\sigma_{g b}$ and grain boundary mobility $\mu_{g b}$ and result in a diffuse grain boundary width $\ell_{g b}$ that is prescribed by numerical (accuracy and stability) and computational (computation time and memory) considerations. 
Combination and rearrangement of equations (13), (29) and (36) gives

$$
\begin{gathered}
\kappa=\sigma_{g b} \ell_{g b} \frac{\sqrt{f_{0, \text { interf }}(\gamma)}}{g(\gamma)} \approx \frac{3}{4} \sigma_{g b} \ell_{g b} \\
L=\frac{\mu_{g b}}{\ell_{g b}} \frac{g(\gamma)}{\sqrt{f_{0}, \operatorname{interf}(\gamma)}} \approx \frac{4}{3} \frac{\mu_{g b}}{\ell_{g b}} \\
m=\frac{\sigma_{g b}}{\ell_{g b}} \frac{1}{g(\gamma) \sqrt{f_{0, \text { interf }}(\gamma)}} \\
\approx \frac{3}{4} \frac{1}{f_{0, \text { saddle }}(\gamma)} \frac{\sigma_{g b}}{\ell_{g b}} .
\end{gathered}
$$

The approximated expressions assume that $g(\gamma) \approx 4 / 3 \sqrt{f_{0, \text { interf }}(\gamma)} \approx 4 / 3 \sqrt{f_{0 \text {,saddle }}(\gamma)}$, which gives very accurate results for $\gamma$-values around 1.5 (see figure 8). If the approximated expressions are not accurate enough, $g(\gamma)$ and $f_{0, \text { interf }}(\gamma)$ can be evaluated from a polynomial fitted through the numerically calculated values of $g(\gamma)$ and $f_{0 \text {,interf }}(\gamma)$. Fifth order polynomials, for example, fit smoothly the calculated data points for $g(\gamma)$ and $f_{0, \text { interf }}(\gamma)$ over a wide range of $\gamma$-values.

To calculate the model parameters, one must first decide on the value of $\gamma$ and determine $g(\gamma)$ and $f_{0, \text { interf }}(\gamma)$. With these values, $\kappa, m$ and $L$ can be calculated from equations (37). In previous grain growth simulations for uniform systems ${ }^{2,6,7,18}, \gamma$ was always taken equal to 1 , giving very satisfactory results. However, the present analysis indicates that $\gamma=1.5$ might be a more appropriate choice, as it results in symmetrical phase field profiles. For symmetrical profiles, the approximations in relations (37) are exact, resulting in simple relations between the model parameters and grain boundary energy and mobility. Moreover, asymmetrical profiles have longer tails for the same grain boundary width (see figure 9); the sharp interface approximation (34) may therefore break down at a larger grain size, although this effect is really small.

If the properties of the system are expressed in SI-units, namely $\sigma_{g b}\left(\mathrm{~J} / \mathrm{m}^{2}\right), \ell_{g b}(\mathrm{~m})$ and $\mu_{g b}\left(\mathrm{~m}^{4} / \mathrm{Js}\right)$, the model parameters have the following dimensions, $\kappa(\mathrm{J} / \mathrm{m}), L\left(\mathrm{~m}^{3} / \mathrm{Js}\right)$ and $m\left(\mathrm{~J} / \mathrm{m}^{3}\right)$. The parameter $\gamma$ is dimensionless.

\section{SYSTEMS WITH NON-UNIFORM GRAIN BOUNDARY PROPERTIES}

In this section, the previous analysis is extended to systems with non-uniform grain boundary properties. The purpose is to derive a methodology for calculating appropriate 
model parameters $\kappa(\theta, \phi), \gamma(\theta, \phi), L(\theta, \phi)$ and $m$, which reproduce accurately a given grain boundary energy $\sigma_{g b}(\theta, \phi)$ and mobility $\mu_{g b}(\theta, \phi)$ as a function of misorientation $\theta$ and grain boundary inclination $\phi$. The model formulation and parameters must guarantee a constant diffuse grain boundary width, in order to resolve the movement of all grain boundaries with an equal accuracy in the numerical simulations. If there would be large variations in grain boundary width with misorientation or inclination, the phase field profiles across thinner grain boundary segments would be less resolved than those across wider segments, while the sharp interface approximation breaks down more easily for wider segments. As a consequence, it would be difficult to control the numerical accuracy of the simulations and artificial effects may be introduced ${ }^{22}$.

\section{A. Model formulation for systems with non-uniform grain boundary properties}

Following the approach of Kazaryan et al. ${ }^{19,20}$, anisotropy is introduced in the model equations (1), (3) and (4) by formulating the parameters $\kappa, \gamma$ and $L$ as a function of the misorientation $\theta$ between adjacent grains and the inclination $\phi$ of the grain boundaries:

$$
\frac{\partial \eta_{i}(\mathbf{r}, t)}{\partial t}=-L(\theta, \phi) \frac{\delta F\left(\eta_{1}, \eta_{2}, \ldots, \eta_{p}\right)}{\delta \eta_{i}(\mathbf{r}, t)}
$$

with

$$
\begin{aligned}
F & =\int_{V}\left[m f_{0}\left(\eta_{1}, \eta_{2}, \ldots, \eta_{p}\right)\right. \\
& \left.+\frac{\kappa(\theta, \phi)}{2} \sum_{i=1}^{p}\left(\nabla \eta_{i}\right)^{2}\right] \mathrm{d} V
\end{aligned}
$$

and

$$
\begin{aligned}
f_{0}\left(\eta_{1}, \eta_{2}, \ldots, \eta_{p}\right) & =\sum_{i=1}^{p}\left(\frac{\eta_{i}^{4}}{4}-\frac{\eta_{i}^{2}}{2}\right) \\
& +\sum_{i=1}^{p} \sum_{j>i}^{p} \gamma(\theta, \phi) \eta_{i}^{2} \eta_{j}^{2}+\frac{1}{4}
\end{aligned}
$$

In the most general case, $\theta$ is a vector with 3 independent coordinates, which define the misorientation between two adjacent grains, and $\phi$ a vector with 2 independent (or 3 normalized) coordinates, which define the inclination of a grain boundary ${ }^{37}$. A difference with the model of Kazaryan et al. and most phase field models for anisotropic systems, is that both the homogeneous free energy and the gradient free energy may be inclination dependent. 
We propose the following misorientation and inclination dependence for the model parameters

$$
\begin{aligned}
\kappa(\theta, \phi) & =\frac{\sum_{i=1}^{p} \sum_{j>i}^{p} \kappa_{i, j}\left(\phi_{i, j}\right) \eta_{i}^{2} \eta_{j}^{2}}{\sum_{i=1}^{p} \sum_{j>i}^{p} \eta_{i}^{2} \eta_{j}^{2}} \\
\gamma(\theta, \phi) & =\frac{\sum_{i=1}^{p} \sum_{j>i}^{p} \gamma_{i, j}\left(\phi_{i, j}\right) \eta_{i}^{2} \eta_{j}^{2}}{\sum_{i=1}^{p} \sum_{j>i}^{p} \eta_{i}^{2} \eta_{j}^{2}}
\end{aligned}
$$

and

$$
L(\theta, \phi)=\frac{\sum_{i=1}^{p} \sum_{j>i}^{p} L_{i, j}\left(\phi_{i, j}\right) \eta_{i}^{2} \eta_{j}^{2}}{\sum_{i=1}^{p} \sum_{j>i}^{p} \eta_{i}^{2} \eta_{j}^{2}} .
$$

The parameter $m$ is constant throughout the system. Misorientation dependence is thus treated in a discrete way, whereas for each misorientation, the parameters are continuous functions of the grain boundary inclination $\phi_{i, j}$. $\phi_{i, j}$ is a normalized vector that specifies the orientation of the normal to the grain boundary between grains with orientations $i$ and $j$, defined as

$$
\phi_{i, j}=\frac{1}{2}\left(\frac{\nabla \eta_{i}}{\left|\nabla \eta_{i}\right|}-\frac{\nabla \eta_{j}}{\left|\nabla \eta_{j}\right|}\right)=\frac{\nabla \eta_{i}-\nabla \eta_{j}}{\left|\nabla \eta_{i}-\nabla \eta_{j}\right|} .
$$

Since the gradients of the two phase field profiles across a grain boundary are always in the same direction, namely perpendicular to the grain boundary surface, but opposite in sign, $\eta_{i}$ and $\eta_{j}$ are treated in an antisymmetric way in the definition for $\phi_{i, j}$. Formulations (41) allow us to specify for each misorientation individually the value and inclination dependence of the grain boundary energy and mobility. Within a diffuse grain boundary region where $\eta_{i}$ and $\eta_{j}$ change values, $\kappa(\theta, \phi)=\kappa_{i, j}\left(\phi_{i, j}\right), \gamma(\theta, \phi)=\gamma_{i, j}\left(\phi_{i, j}\right)$ and $L(\theta, \phi)=L\left(\phi_{i, j}\right)$. Near triple junctions, there is a smooth transition between the parameter functions for the misorientations of the intersecting grain boundaries. With formulation (41b), the homogeneous free energy (40) reduces to

$$
\begin{aligned}
f_{0}\left(\eta_{1}, \eta_{2}, \ldots, \eta_{p}\right) & =\sum_{i=1}^{p}\left(\frac{\eta_{i}^{4}}{4}+\frac{\eta_{i}^{2}}{2}\right) \\
& +\sum_{i=1}^{p} \sum_{j>i}^{p} \gamma_{i, j}\left(\phi_{i, j}\right) \eta_{i}^{2} \eta_{j}^{2}+\frac{1}{4}
\end{aligned}
$$

In principle, there are many possibilities to introduce anisotropy in the free energy functional. An appealing alternative is to assume $\gamma=1.5$ constant and introduce the anisotropy in the homogeneous free energy through the parameter $m$ and $\kappa$, with

$$
m(\theta, \phi)=\frac{\sum_{i=1}^{p} \sum_{j>i}^{p} m_{i, j}\left(\phi_{i, j}\right) \eta_{i}^{2} \eta_{j}^{2}}{\sum_{i=1}^{p} \sum_{j>i}^{p} \eta_{i}^{2} \eta_{j}^{2}}
$$


and $\kappa$ as given in (41a). The advantage is that the phase field profiles are symmetrical for all grain boundaries. However, application of this model showed that an impractical high numerical resolution (fine grid spacing) is required to obtain correct equilibrium angles between intersecting grain boundaries at triple junctions.

\section{B. Misorientation dependence}

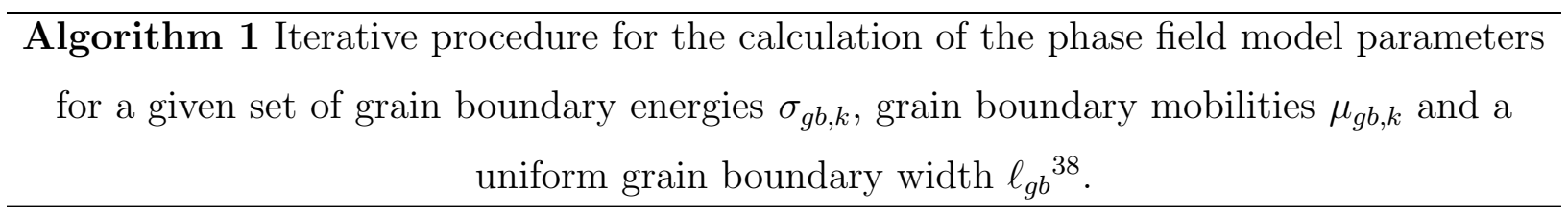

Input:

$\left\{\sigma_{g b, k}\right\},\left\{\mu_{g b, k}\right\}, \ell_{g b}$

$\sigma_{g b, \text { init }}, \gamma_{\text {init }}$

Initial Step: Calculate $g\left(\gamma_{\text {init }}\right), f_{0, \text { interf }}\left(\gamma_{\text {init }}\right)$ and $a_{\text {init }}=\sqrt{f_{0, \text { interf }}\left(\gamma_{\text {init }}\right)} / g\left(\gamma_{\text {init }}\right)$

Calculate $m$ from equation (37c) using $\sigma_{g b, \text { init }}, \ell_{g b}, f_{0, \text { interf }}\left(\gamma_{\text {init }}\right)$ and $g\left(\gamma_{\text {init }}\right)$

Body:

For each $k$ :

Iterative calculation of $\kappa_{k}, \gamma_{k}$ and $a_{k}$

1. Calculate $\kappa^{*}=\sigma_{g b, k} \ell_{g b} a_{\text {init }}$

2. Calculate $g\left(\gamma^{*}\right)=\sigma_{g b, k} / \sqrt{\kappa^{*} m}$

3. Calculate $\gamma^{*}=g^{-1}$

4. Calculate $f_{0, \text { interf }}\left(\gamma^{*}\right)$ and $a_{k}^{*}=\sqrt{f_{0, \text { interf }}\left(\gamma^{*}\right)} / g\left(\gamma^{*}\right)$

5. If $a_{k}^{*} \neq a_{\text {init }}, a_{\text {init }}=a_{k}^{*}$ and go to 1 ., else $\kappa_{k}=\kappa^{*}, \gamma_{k}=\gamma^{*}, a_{k}=a^{*}$

Calculate $L_{k}$ from equation (37b) using $\mu_{g b, k}, \ell_{g b}$ and $a_{k}$

Output:

$m,\left\{\kappa_{k}\right\},\left\{\gamma_{k}\right\},\left\{L_{k}\right\}$ 
If inclination dependence is neglected, it follows from the formulation (41) that $\kappa(\theta)=$ $\kappa_{i, j}, \gamma(\theta)=\gamma_{i, j}$ and $L(\theta)=L_{i, j}$ are constant within the diffuse grain boundary region between grains with orientations $i$ and $j$. By consequence, relations (37) apply for an individual grain boundary. One difficulty is that now, there are different $\gamma_{i, j}$ 's for grain boundaries with different energies. These values must be chosen so that each grain boundary has the correct energy, while all grain boundaries have the same width. Due to the complicated relations between the model parameter $\gamma_{i, j}$ and the grain boundary properties, an iterative procedure, described by algorithm 1 , is required to obtain the $\gamma_{i, j}$ for different misorientations.

Algorithm 1 requires, as input, lists of discrete values $\sigma_{g b, k}$ and $\mu_{g b, k}$, the desired grain boundary width $\ell_{g b}$ and two initial values $\gamma_{\text {init }}$ and $\sigma_{g b \text {,init }}$. In the first step, the parameter $m$, which is uniform throughout the system, is calculated using the initial values $\gamma_{\text {init }}$ and

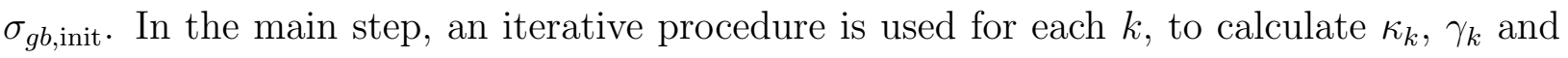
$L_{k}$. To start, a first estimate $\kappa^{*}$ and $\gamma^{*}$ is made based on $a_{\text {init }}$ calculated for $\gamma_{\text {init }}$ or the $a_{k}$ calculated for the previous misorientation. A new value $a_{k}^{*}$, is also computed for $\kappa^{*}$ and $\gamma^{*}$. If $a_{k}^{*} \neq a_{\text {init }}$, improved values for $\kappa^{*}$ and $\gamma^{*}$ are calculated based on updated values for $a_{k}^{*}$ in subsequent iteration steps, until the values for $a_{k}^{*}, \gamma^{*}$ and $\kappa^{*}$ are self-consistent. Then, the kinetic parameter $L_{k}$ is calculated using the self-consistent value for $a_{k}$. The output of the procedure are the parameter $m$ and a list with the appropriate values for $\kappa_{k}, \gamma_{k}$ and $L_{k}$.

To be able to calculate $g\left(\gamma_{k}\right), \gamma_{k}=g^{-1}\left(\gamma_{k}\right)$ and $f_{0, \text { interf }}\left(\gamma_{k}\right)$ for arbitrary values of $\gamma_{k}$, the numerically calculated function values for $f_{0, \text { interf }}(1 / \gamma), g^{2}(1 / \gamma)$ and $\left(g^{2}\right)^{-1}(1 / \gamma)$ were interpolated with fifth order polynomials for $\gamma$ within the range $\left[\begin{array}{ll}0.55 & 8\end{array}\right]$. In step 3 of the algorithm, $\gamma^{*}=g^{-1}$ is calculated via the interpolated function $g^{2}(1 / \gamma): g \rightarrow g^{2} \rightarrow 1 / \gamma^{*} \rightarrow$ $\gamma^{*}$. If all $\gamma_{k}$ can be taken close to 1.5 , the iterative procedure may be avoided by assuming that $g(\gamma)=(4 / 3) * \sqrt{f_{0, \text { interf }}}=(4 / 3) * \sqrt{f_{0, \text { saddle }}}$.

At the beginning of the procedure, it is arbitrarily chosen that $\gamma\left(\theta_{\text {init }}\right)=\gamma_{\text {init. }}$. The parameter values for the other misorientations follow from this choice. For different initial values, a different set of model parameters is obtained, which results in the same grain boundary energies, mobilities and uniform grain boundary width. We advice to choose $\gamma_{\text {init }}$ around 1.5 for a $\theta_{\text {init }}$ within the range of the $\theta_{k}$-values, in order to obtain close to symmetrical grain boundary profiles.

As the function $g(\gamma)$ flattens for larger values of $\gamma$, there is a practical limit on the ratio $\sigma_{\max } / \sigma_{\min }$ that can be covered by the model. From the numerically calculated data for 
$g\left(\gamma_{i, j}\right)$, it is derived that for $\gamma$ in the range $\left[\begin{array}{ll}0.9 & 2.65\end{array}\right], g\left(\gamma_{i, j}\right) \in\left[\begin{array}{ll}0.36 & 0.56\end{array}\right]$ and $\sigma_{\max } / \sigma_{\min }$ $=g_{\max }^{2} / g_{\min }^{2}=2.38$, for $\gamma$ in the range $\left[\begin{array}{ll}0.75 & 3.45\end{array}\right], g\left(\gamma_{i, j}\right) \in\left[\begin{array}{ll}0.30 & 0.59\end{array}\right]$ and $\sigma_{\max } / \sigma_{\min }$ $=g_{\max }^{2} / g_{\min }^{2}=3.73$ and for $\gamma$ in the range $\left[\begin{array}{ll}0.53 & 6.5\end{array}\right], g\left(\gamma_{i, j}\right) \in\left[\begin{array}{ll}0.12 & 0.65\end{array}\right], \sigma_{\max } / \sigma_{\min }$ $=g_{\max }^{2} / g_{\min }^{2}=29.4$ and $\gamma$ in the range $\left[\begin{array}{ll}0.52 & 8\end{array}\right], g\left(\gamma_{i, j}\right) \in\left[\begin{array}{ll}0.0985 & 0.67\end{array}\right], \sigma_{\max } / \sigma_{\min }=$ $g_{\max }^{2} / g_{\min }^{2}=45.8$. The latter may be considered as a practical limit.

\section{Inclination dependence}

For a given inclination $\phi_{i, j}$, the parameters $\kappa_{i, j}\left(\phi_{i, j}\right), \gamma_{i, j}\left(\phi_{i, j}\right), L_{i, j}\left(\phi_{i, j}\right)$ and $m$ are constant along the normal to the grain boundary. In appendix A, it is explained how to extend the asymptotic analysis described in Refs. ${ }^{34-36}$ to the current phase field model (38)-(40). It is derived that the profiles of the phase field variables across a grain boundary satisfy equations (7) to leading order for $\ell_{g b} \ll R_{1}, R_{2}$, with $\mathrm{x}$ measured along the normal to the surface and $\kappa_{i, j}=\kappa_{i, j}\left(\phi_{i, j}\right)$ and $\gamma_{i, j}=\gamma_{i, j}\left(\phi_{i, j}\right)$ constant along the profiles. Consequently, relations (13) and (29), with $g\left(\gamma_{i, j}\right)$ and $f_{0, \text { interf }}\left(\gamma_{i, j}\right)$ as calculated for uniform systems, apply along each direction $\phi_{i, j}$, giving

$$
\sigma_{g b}\left(\theta_{i, j}, \phi\right)=g\left(\gamma_{i, j}\left(\phi_{i, j}\right)\right) \sqrt{\kappa_{i, j}\left(\phi_{i, j}\right) m}
$$

and

$$
\ell_{g b}=\sqrt{\frac{\kappa_{i, j}\left(\phi_{i, j}\right)}{m f_{0, \text { interf }}\left(\gamma_{i, j}\left(\phi_{i, j}\right)\right)}},
$$

in which $\sigma_{g b}\left(\theta_{i, j}, \phi\right)$ refers to the inclination dependent specific energy of a grain boundary between the grains with orientations $i$ and $j$. Furthermore, it is obtained that in the sharp interface limit, the normal velocity of each point on a grain boundary equals

$$
\begin{array}{r}
v_{g b, n}=-L_{i, j}\left(\phi_{i, j}\right)\left[\frac{1}{R_{1}}\left(\kappa_{i, j}\left(\phi_{i, j}\right)+\frac{\partial^{2} \kappa_{i, j}\left(\phi_{i, j}\right)}{\partial \alpha_{1}^{2}}\right)\right. \\
\left.+\frac{1}{R_{2}}\left(\kappa_{i, j}\left(\phi_{i, j}\right)+\frac{\partial^{2} \kappa_{i, j}\left(\phi_{i, j}\right)}{\partial \alpha_{2}^{2}}\right)\right]
\end{array}
$$

with $\phi_{i, j}$ the normal direction and $R_{1}, R_{2}, \alpha_{1}$ and $\alpha_{2}$ defined in a curvilinear coordinate system as shown in figure 1b. For sharp interface systems with inclination dependence, Herring's formula ${ }^{39}$ prescribes a normal grain boundary velocity $v_{g b, n}$

$$
\begin{array}{r}
v_{g b, n}=-\mu_{g b}(\phi)\left[\frac{1}{R_{1}}\left(\sigma_{g b}(\phi)+\frac{\partial^{2} \sigma_{g b}(\phi)}{\partial \alpha_{1}^{2}}\right)\right. \\
\left.+\frac{1}{R_{2}}\left(\sigma_{g b}(\phi)+\frac{\partial^{2} \sigma_{g b}(\phi)}{\partial \alpha_{2}^{2}}\right)\right],
\end{array}
$$




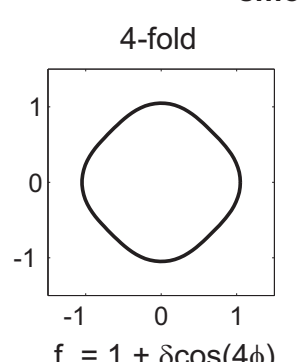

with $\delta=0.05$

a) $\quad f_{\sigma}^{\max } / f_{\sigma}^{\min }=1.10$

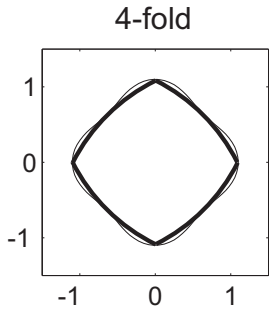

$\mathrm{f}_{\sigma}=1+\delta \cos (4 \phi)$,

with $\delta=0.1$

b) $\quad f_{\sigma}^{\max } / f_{\sigma}^{\min }=1.22$

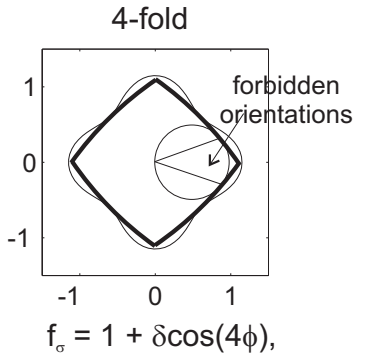

with $\delta=0.15$

c) $\quad f_{\sigma}^{\max } / f_{\sigma}^{\min }=1.35$

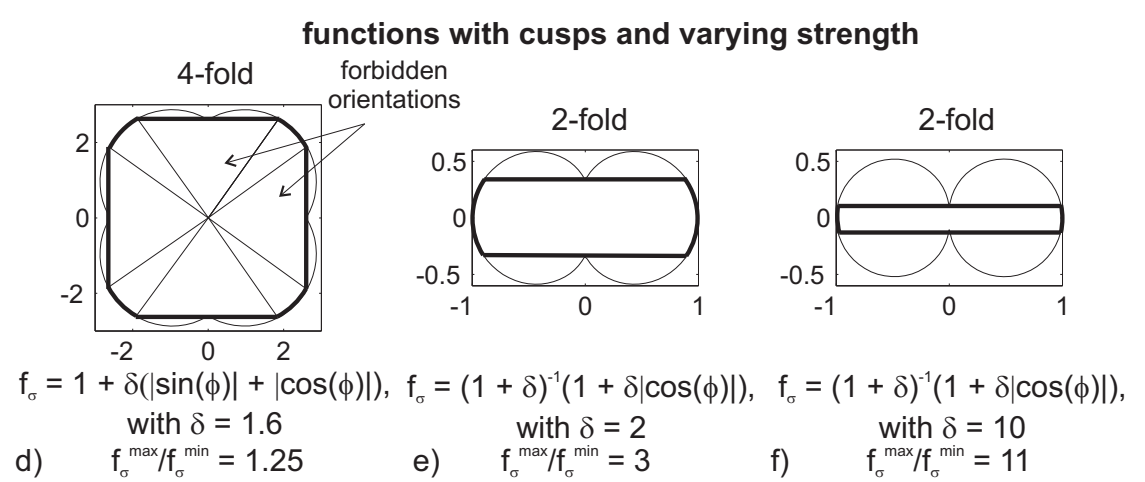

FIG. 11: Polar plots of a number of inclination dependent functions $f_{\sigma}(\phi)$ for $2 \mathrm{D}$ systems. The equilibrium shape of the crystal, determined according to Wulff's theorem ${ }^{40}$, is plotted in bold. It only contains those orientations for which $f_{\sigma}(\phi)+\mathrm{d} f_{\sigma}(\phi) / \mathrm{d} \phi>0$. For simulations, the non-convex parts must be regularized as described in Refs. ${ }^{41-43}$.

where $\sigma_{g b}(\phi)+\frac{\partial^{2} \sigma_{g b}(\phi)}{\partial \alpha_{2}^{2}}$ is called the grain boundary stiffness. Comparison of (47) and (48) shows that $\kappa_{i, j}\left(\phi_{i, j}\right)$ must have the same inclination dependent factor as the specific grain boundary energy $\sigma_{g b}\left(\theta_{i, j}, \phi\right)$, to obtain equal velocities. For instance, if $\sigma_{g b}\left(\theta_{i, j}, \phi\right)$ has the form $\bar{\sigma}_{g b} f_{\sigma}(\phi), \kappa_{i, j}\left(\phi_{i, j}\right)$ must have the form $\bar{\kappa}_{i, j} f_{\sigma}\left(\phi_{i, j}\right)$, with $f_{\sigma}\left(\phi_{i, j}\right)=f_{\sigma}(\phi)$. If $\kappa_{i, j}$ has the same inclination dependence as the grain boundary energy, relation (36) remains valid for individual directions $\phi_{i, j}$, giving

$$
\mu_{g b}\left(\theta_{i, j}, \phi\right) \sigma_{g b}\left(\theta_{i, j}, \phi\right)=\kappa_{i, j}\left(\phi_{i, j}\right) L_{i, j}\left(\phi_{i, j}\right)
$$

with $\sigma_{g b}\left(\theta_{i, j}, \phi\right)$ and $\mu_{g b}\left(\theta_{i, j}, \phi\right)$ defined as for relation (45).

To formulate the inclination dependent model parameters $\kappa_{i, j}\left(\phi_{i, j}\right), \gamma_{i, j}\left(\phi_{i, j}\right)$ and $L_{i, j}\left(\phi_{i, j}\right)$, it is assumed that the grain boundary properties of the system are characterized by a set of inclination dependent grain boundary energies $\sigma_{g b, k}(\phi)=\bar{\sigma}_{g b, k} f_{\sigma, k}(\phi)$ and mobilities $\mu_{g b, k}(\phi)=\bar{\mu}_{g b, k} f_{\mu, k}(\phi)$, where $k$ refers to different misorientations (the difference in 
orientation between grains with orientations $i$ and $j$, for different combinations of $i$ and $j$ ). The grain boundary energies and mobilities may have a different inclination dependence and the inclination dependence may vary with misorientation. Furthermore, 2 different approaches are proposed for the two extreme cases of weak and strong inclination dependence of the grain boundary energy, resulting in respectively smooth and faceted grain boundaries. To support the explanation, a number of inclination dependent factors $f_{\sigma}(\phi)$ with different shapes and strengths, typically used for 2D systems, are shown in figure 11. More functions, also for 3D systems, are available in the literature ${ }^{44,45}$. Intermediate cases, as for example plots c and e in figure 11, can be treated by both approaches. Since grain growth may be considered as a slow process and attachment kinetics in metals are relatively fast, it is assumed that the shape of the crystals is primarily determined by the inclination dependence of the grain boundary energy.

\section{Smooth grain boundaries}

When the inclination dependence of the specific grain boundary energy is smooth, all or most grain boundary inclinations may occur from a thermodynamic point of view. The set of model parameters must thus reflect the full inclination dependence of the specific grain boundary energy, stiffness and mobility, to obtain correct grain boundary behavior in the simulations. Therefore, it follows from comparison of relations (47) and (48) that $\kappa_{k}$ must be of the form

$$
\kappa_{k}\left(\phi_{k}\right)=\bar{\kappa}_{k} f_{\sigma, k}\left(\phi_{k}\right)
$$

to reproduce grain boundary stiffness. Furthermore, the relations (45) and (49) give that

$$
L_{k}\left(\phi_{k}\right)=\bar{L}_{k} f_{\mu, k}\left(\phi_{k}\right)
$$

and

$$
\begin{aligned}
& \gamma_{k}\left(\phi_{k}\right)=g^{-1}\left(\gamma_{k}\right) \quad \text { with } \\
& g^{2}\left(\gamma_{k}\right)=g^{2}\left(\bar{\gamma}_{k}\right) f_{\sigma, k}\left(\phi_{k}\right)
\end{aligned}
$$

to reproduce the given inclination dependent grain boundary energies and mobilities, and fulfill requirements (A6) and (A7). The values for $m, \bar{\gamma}_{k}, g^{2}\left(\bar{\gamma}_{k}\right), \bar{\kappa}_{k}$, and $\bar{L}_{k}$ can be calculated 
for each misorientation $\theta_{k}$ with algorithm 1 , using the given $\bar{\sigma}_{g b, k}, \bar{\mu}_{g b, k}$ and an appropriate value for $\ell_{g b}$ as input data.

For $\gamma_{k}$ not too far from 1.5, $g\left(\gamma_{k}\right)$ is approximated closely by the analytical function for $4 / 3 \sqrt{f_{0, \text { saddle }}\left(\gamma_{k}\right)}$ (see figure 8). Then, a differentiable function for $\gamma_{k}\left(\phi_{k}\right)$

$$
\gamma_{k}\left(\phi_{k}\right)=\frac{-\frac{9}{4} g^{2}(\bar{\gamma}) f_{\sigma, k}\left(\phi_{k}\right)-1}{\frac{9}{2} g^{2}(\bar{\gamma}) f_{\sigma, k}\left(\phi_{k}\right)-2}
$$

can be obtained from relation (16). More generally, differentiable functions for the inclination dependence of $\gamma_{k}\left(\phi_{k}\right)$ can be obtained by fitting polynomials through the numerically calculated data points for $g\left(\gamma_{k}\right)$ within the ranges of $g\left(\gamma_{k}\right)$-values of interest, namely those covering $f_{\sigma}^{\min }$ and $f_{\sigma}^{\max }$. However, for large ratios $f_{\sigma}^{\max } / f_{\sigma}^{\min }$, the procedure for faceted grain boundaries is probably more appropriate.

In order to obtain parameter functions that are differentiable with respect to grain boundary inclination, the ratio $a_{k}=g\left(\gamma_{k}\right) / \sqrt{f_{0, \max }\left(\gamma_{k}\right)}$ is assumed to be constant along a grain boundary with fixed misorientation, namely $a_{k}=g\left(\bar{\gamma}_{k}\right) / \sqrt{f_{0, \max }\left(\bar{\gamma}_{k}\right)}$. Still, variations in grain boundary width with inclination will be negligible for weak and moderate inclination dependence of the specific grain boundary energy, since the $\gamma$-dependence of $a_{k}$ may be considered as constant within limited ranges of $\gamma_{k}$-values. For $\gamma_{k}$ varying between 0.9 and 2.65, the deviations in grain boundary width are smaller than $0.81 \%$; for $\gamma_{k}$ between 0.75 and 3.45 , they are below $1.6 \%$. The highest accuracy is expected for a set of model parameters with, as far as possible, all $\bar{\gamma}_{k}$-values around 1.5 .

In most phase field models, the inclination dependence of the grain boundary energy is completely treated in the gradient term of the free energy functional, resulting in straightforward inclination dependent relations between the model parameters and the grain boundary energy. However, in this way the variations in grain boundary width with inclination are linearly proportional with the variations in grain boundary energy. Moreover, in these models the inclination dependence of the gradient term contributes to the inclination dependence of the grain boundary mobility. In relations (50), by contrast, the inclination dependence of the kinetic coefficient is directly related to that of the grain boundary mobility. 


\section{Faceted grain boundaries}

In the case of strong anisotropy, there are large variations in specific grain boundary energy with inclination. The inclination dependent parameter functions (50) may then result in variations in grain boundary width with inclination and more complicated functions would be required to interpolate $g^{-1}(\gamma)$ over a wider range of $\gamma_{k}$-values in (50c). Moreover, due to strong variations in $\sigma_{g b}$ with inclination, only a limited number of discrete inclinations, or very narrow inclination ranges, are thermodynamically stable, namely those for which $f_{\sigma}(\phi)+\mathrm{d} f_{\sigma}(\phi) / \mathrm{d} \phi>0$. The plots $\mathrm{d}$-f in figure 11 also show that the crystal shape is mainly determined by the extrema of the inclination dependent factor $f_{\sigma}(\phi)$ and that within a range of stable inclinations, the variation of $f_{\sigma}(\phi)$ is very limited. It is actually most important that the parameters in the phase field model reproduce accurately the extrema of the inclination dependent factor. In the case of strong inclination dependence, the following procedure is accordingly more appropriate to determine inclination dependent functions for the model parameters in the phase field model.

First, a set of discrete values $\kappa_{k, l}, \gamma_{k, l}, L_{k, l}$ and $m$ is calculated using algorithm 1 with a list of $\sigma_{g b}\left(\theta_{k}, \phi_{k, l}\right)$ and $\mu_{g b}\left(\theta_{k}, \phi_{k, l}\right)$ values and an appropriate grain boundary width $\ell_{g b}$ as input. The $\phi_{k, l}$ are discrete inclinations for which $f_{\sigma, k}\left(\phi_{k}\right)$ is extremal. The iterative calculation in the body of algorithm 1 is now performed for each extremal inclination $\phi_{k, l}$ for each misorientation $\theta_{k}$. The inclination dependent model parameters, for each misorientation $k$, are subsequently formulated as

$$
\begin{gathered}
\kappa_{k}\left(\phi_{k}\right)=\bar{\kappa}_{k} f_{\sigma, k}^{\prime}\left(\phi_{k}\right) \\
\gamma_{k}\left(\phi_{k}\right)=\bar{\gamma}_{k} f_{\sigma, k}^{\prime \prime}\left(\phi_{k}\right) \\
L_{k}\left(\phi_{k}\right)=\bar{L}_{k} f_{\mu, k}\left(\phi_{k}\right) .
\end{gathered}
$$

The functions $f_{\sigma, k}^{\prime}\left(\phi_{k}\right)$ and $f_{\sigma, k}^{\prime \prime}\left(\phi_{k}\right)$ have cusps and extrema at the same inclinations as $f_{\sigma, k}\left(\phi_{k}\right)$, the inclination dependent factor of the specific grain boundary energy. Furthermore, the values of $\bar{\kappa}_{k}$ and $\bar{\gamma}_{k}$ are taken so that the extremal values of $\bar{\kappa}_{k} f_{\sigma, k}^{\prime}\left(\phi_{k}\right)$ and $\bar{\gamma}_{k} f_{\sigma, k}^{\prime \prime}\left(\phi_{k}\right)$ equal the $\kappa_{k, l}$ and $\gamma_{k, l}$ calculated in the first step for the extremal values of the specific grain boundary energies. An easy way to fulfill these requirements, is to give $f_{\sigma, k}^{\prime}$ and $f_{\sigma, k}^{\prime \prime}$ a similar 
form as $f_{\sigma, k}$, with, for example, for the function plotted in figure 11d,

$$
\begin{gathered}
\delta_{k}^{\prime}=\frac{\kappa_{\max } / \kappa_{\min }-1}{\sqrt{2}-\kappa_{\max } / \kappa_{\min }} \\
\bar{\kappa}_{k}=\frac{\kappa_{\max }}{1+\sqrt{2} \delta_{k}^{\prime}}=\frac{\kappa_{\min }}{1+\delta_{k}^{\prime}}
\end{gathered}
$$

or for the functions plotted in figures $11 \mathrm{e}-\mathrm{f}$

$$
\begin{gathered}
\delta_{k}^{\prime}=\kappa_{\max } / \kappa_{\min }-1 \\
\bar{\kappa}_{k}=\kappa_{\max }=\kappa_{\min }\left(1+\delta_{k}^{\prime}\right) .
\end{gathered}
$$

In both cases $\kappa_{\max }$ and $\kappa_{\min }$ are the $\kappa_{k, l}$ calculated for the inclination $l$ for which the specific grain boundary energy is maximal or minimal. As the range of stable inclinations and the variation of the specific grain boundary energy within these ranges are extremely small and primarily determined by the extrema, the inclination dependence of the homogeneous free energy is formulated directly for the parameter $\gamma$.

\section{Force balance at edges and multi junctions}

For a number of phase field models it has been verified that in the limit of sharp interfaces $\left(\ell_{g b} \ll R_{1}, R_{2}\right)$, surface stress is balanced at edges and triple junctions ${ }^{35,46}$. In appendix A, the same analysis is applied to the present model. A result is that, the force, due to surface stress, of a grain boundary between grains $i$ and $j$, acting on a plane that cuts the boundary perpendicular, is given by

$$
\begin{aligned}
f & =\int_{0}^{\infty}\left[\left(\frac{\partial \eta_{i}}{\partial r}\right)^{2}+\left(\frac{\partial \eta_{j}}{\partial r}\right)^{2}\right] \mathrm{d} r\left(\frac{\partial \kappa_{i, j}}{\partial \alpha_{1}} \mathbf{r}-\kappa_{i, j} \mathbf{t}_{1}\right) \\
& =\frac{\sigma_{g b}}{\kappa_{i, j}} \frac{\partial \kappa_{i, j}}{\partial \alpha_{1}} \mathbf{r}-\sigma_{g b} \mathbf{t}_{1},
\end{aligned}
$$

with $\mathbf{r}, \mathbf{t}_{1}$ and $\mathbf{t}_{2}$ curvilinear, $\mathbf{t}_{1}$ perpendicular to the plane and $\mathbf{t}_{2}$ common to the plane and the grain boundary surface. The relation assumes that $\ell_{g b} \ll R_{1}, R_{2}$. If, furthermore, $\kappa_{i, j}$ has the same orientation dependence as $\sigma_{g b}$,

$$
f=\frac{\partial \sigma_{g b}}{\partial \alpha_{1}} \mathbf{r}-\sigma_{g b} \mathbf{t}_{1}
$$

is obtained. The tangential term drives the grain boundary to move towards its center of curvature to shorten its length. The radial term is a torque that forces the grain boundary to reorient itself towards an orientation with lower energy. 
Considering a closed surface that cuts perpendicular all grain boundaries meeting at an edge or multi-junction, application of Neother's theorem subsequently gives that

$$
\sum_{k} \frac{\partial \sigma_{g b}\left(\theta_{k}, \alpha_{1, k}\right)}{\partial \alpha_{1, k}} \mathbf{r}_{k}-\sigma_{g b}\left(\theta_{k}, \alpha_{1, k}\right) \mathbf{t}_{1, k}=0,
$$

where the sum is taken over all grain boundary segments that meet at the junction. $r_{k}$ and $t_{1, k}$ are the radial and tangential curvilinear coordinate for grain boundary $k, \alpha_{1, k}$ the inclination angle measured in the plane $\left(\mathbf{r}_{k}, \mathbf{t}_{1, k}\right)$. Furthermore, the intersection line of the grain boundaries is directed along $\mathbf{t}_{2,1}=\mathbf{t}_{2,2}=\mathbf{t}_{2, k}$. Equation (55) is exactly the force balance requirement for edges and multi-junctions obtained in sharp interface models ${ }^{40,47}$.

In table I, triple junction angles obtained from numerical simulations are listed for 3 different combinations of grain boundary energies. Junctions in equilibrium and in steadystate motion were considered for 3 different combinations of energies of the intersecting grain boundaries. For $\beta_{c} \geq 120^{\circ}$, the theoretical values $\beta_{c}$ for triple junctions in equilibrium (Y) are very well reproduced when $\ell_{g b} / \Delta x>5$, which is not more restrictive as the requirement for accurate curvature driven grain boundary movement (see section II G). Those obtained during steady-state motion are slightly smaller than the equilibrium angles. As this deviation increases with grain boundary width, it may be devoted to the diffuse character of the boundaries. Probably, at and near the triple junction, the two curved boundaries attract each other from their outside. When 2 lower-energy boundaries meet with a higher-energy boundary, a $\beta_{c}<120^{\circ}$ is expected. In this case, the theoretical value for $\beta_{c}$ is less well reproduced, also for the equilibrium structure. The deviation increases for smaller angles (increasing ratio $\left.\sigma_{g b, \max } / \sigma_{g b, \min }\right)$. It is not clear why and how to reduce the difference. However, we must remark that a situation where two boundaries with a low energy meet a boundary with a considerably higher energy is rare in polycrystalline structures.

\section{E. Difficulties of a fully variational approach with respect to misorientation de-}

\section{pendence}

Since $\kappa$ is a function of the phase field variables and their gradients, in a fully variational approach, the contribution from the gradient energy in the thermodynamic driving forces ${ }^{48}$ 
TABLE I: Triple junction angles as obtained in simulations for a triple junction in equilibrium (Y) and one in steady-state movement $(\mathrm{T})$. The effect of grain boundary width $\ell_{g b}$ and grid spacing $\Delta x$ was studied for three different configurations, with a theoretically expected value for the angle $\beta_{c}$ equal to $102,6^{\circ}, 120^{\circ}$ and $138,15^{\circ}$.

\begin{tabular}{|c|c|c|c|}
\hline Geometries & Equilibrium & Stead & $y$ state $(Y)$ \\
\hline Theory: & & & \\
\hline Young's law & $\beta_{c}=102,60^{\circ}$ & $\beta_{c}=120,00^{\circ}$ & $\beta_{c}=138,15^{\circ}$ \\
\hline$\Delta x=0.2, \ell_{g b}=0.5$ & $110,0(\mathrm{Y})$ & $121,3(\mathrm{Y})$ & $140,2(\mathrm{Y})$ \\
\hline & $107,5(\mathrm{~T})$ & $119,8(\mathrm{~T})$ & $139,9(\mathrm{~T})$ \\
\hline$\Delta x=0.2, \ell_{g b}=1$ & $109.1(\mathrm{Y})$ & $120,3(\mathrm{Y})$ & $139,2(\mathrm{Y})$ \\
\hline & $103,8(\mathrm{~T})$ & $117,3(\mathrm{~T})$ & $138,3(\mathrm{~T})$ \\
\hline$\Delta x=0.1, \ell_{g b}=0.5$ & $109,1(\mathrm{Y})$ & $120,3(\mathrm{Y})$ & $139,2(\mathrm{Y})$ \\
\hline & $105,7(\mathrm{~T})$ & $118,4(\mathrm{~T})$ & $138,7(\mathrm{~T})$ \\
\hline$\Delta x=0.1, \ell_{g b}=1$ & $108,8(\mathrm{Y})$ & $120,3(\mathrm{Y})$ & $139,0(\mathrm{Y})$ \\
\hline & $104,7(\mathrm{~T})$ & $117.6(\mathrm{~T})$ & $138,2(\mathrm{~T})$ \\
\hline$\Delta x=0.1, \ell_{g b}=2$ & $108,8(\mathrm{Y})$ & $120,3(\mathrm{Y})$ & $139,2(\mathrm{Y})$ \\
\hline & $103,3(\mathrm{~T})$ & $116.6(\mathrm{~T})$ & $136,7(\mathrm{~T})$ \\
\hline
\end{tabular}

$\partial F / \partial \eta_{i}$ should be calculated $\operatorname{as}^{32}$

$$
\begin{aligned}
& \frac{\delta}{\delta \eta_{i}} \int_{V} {\left[\frac{\kappa}{2} \sum_{j=1}^{p}\left(\nabla \eta_{j}\right)^{2}\right] \mathrm{d} V=\frac{1}{2}\left(\frac{\partial \kappa}{\partial \eta_{i}}\right) \sum_{j=1}^{p}\left(\nabla \eta_{j}\right)^{2} } \\
&-\nabla \cdot\left[\frac{1}{2}\left(\frac{\partial \kappa}{\partial \nabla \eta_{i}}\right) \sum_{j=1}^{p}\left(\nabla \eta_{j}\right)^{2}+\kappa \nabla \eta_{i}\right]
\end{aligned}
$$


with

$$
\begin{gathered}
-\nabla \cdot\left[\frac{1}{2}\left(\frac{\partial \kappa}{\partial \nabla \eta_{i}}\right) \sum_{j=1}^{p}\left(\nabla \eta_{j}\right)^{2}\right] \\
=-\frac{1}{2} \sum_{j=1}^{p}\left(\nabla \eta_{j}\right)^{2} \nabla \cdot\left[\frac{\partial \kappa}{\partial \nabla \eta_{i}}\right] \\
-\frac{1}{2} \frac{\partial \kappa}{\partial \nabla \eta_{i}} \cdot \nabla\left[\sum_{j=1}^{p}\left(\nabla \eta_{j}\right)^{2}\right], \\
-\nabla \cdot \frac{\partial \kappa}{\partial \nabla \eta_{i}}=-\nabla \cdot \sum_{j \neq i}^{p}\left[\frac{\partial \kappa}{\partial \phi_{i, j}} \frac{\partial \phi_{i, j}}{\partial \nabla \eta_{i}}\right] \\
=-\sum_{j \neq i}^{p}\left[\left(\frac{\partial^{2} \kappa}{\partial \phi_{i, j}^{2}} \nabla \phi_{i, j}+\sum_{k=1}^{p} \frac{\partial^{2} \kappa}{\partial \eta_{k} \partial \phi_{i, j}} \nabla \eta_{k}\right) \cdot \frac{\partial \phi_{i, j}}{\partial \nabla \eta_{i}}\right] \\
-\sum_{j \neq i}^{p} \frac{\partial \kappa}{\partial \phi_{i, j}} \nabla \cdot\left[\frac{\partial \phi_{i, j}}{\partial \nabla \eta_{i}}\right],
\end{gathered}
$$

and

$$
\begin{aligned}
-\nabla \cdot\left[\kappa \nabla \eta_{i}\right]=-(\nabla \kappa) \cdot\left(\nabla \eta_{i}\right)-\kappa \nabla^{2} \eta_{i} \\
=-\sum_{j=1}^{p}\left(\frac{\partial \kappa}{\partial \eta_{j}}\right)\left(\nabla \eta_{j}\right) \cdot\left(\nabla \eta_{i}\right) \\
\quad-\sum_{j=1}^{p} \sum_{k \neq j}^{p}\left(\frac{\partial \kappa}{\partial \phi_{j, k}}\right)\left(\nabla \phi_{j, k}\right) \cdot\left(\nabla \eta_{i}\right)-\kappa \nabla^{2} \eta_{i} .
\end{aligned}
$$

The derivatives of $\kappa_{i, j}$ with respect to the gradients of the phase field variables, $\partial \kappa_{i, j} / \partial \nabla \eta_{i}$, give rise to a driving force that rotates the grain boundary towards a direction with lower energy, which is observed on a mesoscale for metals.

The derivatives of $\kappa$ with respect to the phase field variables, $\partial \kappa / \partial \eta_{i}$, result in a driving force that changes the local misorientation towards one with a lower energy, by changing the local values of the phase field variables. For instance, if $\kappa_{i, k}=\kappa_{j, k}<\kappa_{i, j}$, the value of $\kappa$ in the diffuse grain boundary region between grains $i$ and $j$ is lowered when the phase field variable $\eta_{k}(\mathrm{x})$ takes a finite value at the interface, since equation (41a) then gives

$$
\kappa=\frac{\kappa_{i, j} \eta_{i}^{2} \eta_{j}^{2}+\kappa_{i, k} \eta_{i}^{2} \eta_{k}^{2}+\kappa_{j, k} \eta_{j}^{2} \eta_{k}^{2}}{\eta_{i}^{2} \eta_{j}^{2}+\eta_{i}^{2} \eta_{k}^{2}+\eta_{j}^{2} \eta_{k}^{2}}<\frac{\kappa_{i, j} \eta_{i}^{2} \eta_{j}^{2}}{\eta_{i}^{2} \eta_{j}^{2}}=\kappa_{i, j} .
$$


a)
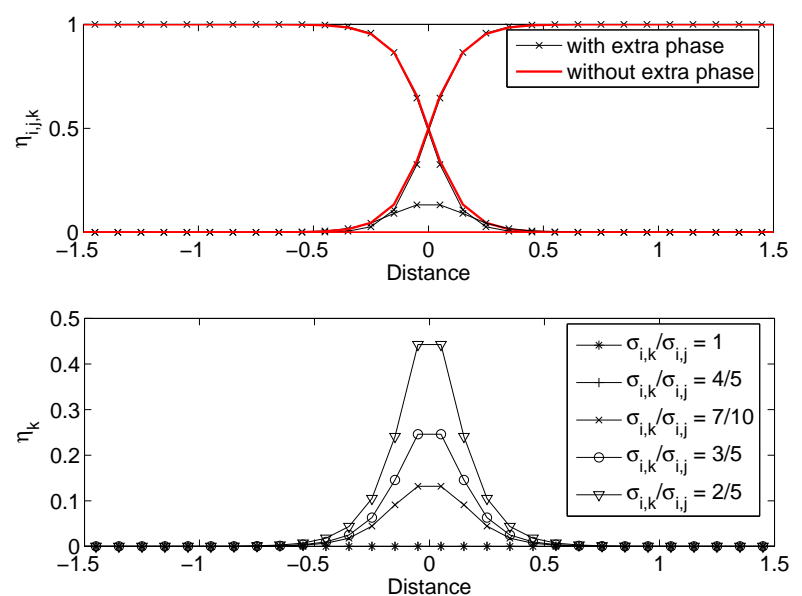

FIG. 12: Simulation results that are obtained when the $\eta$-dependence of the parameter $\kappa$ is considered in the driving force: a) effect of an extra phase field variable, $\eta_{k} \neq 0$, on the profiles of the phase field variables $\eta_{i}$ and $\eta_{j}$ for $\sigma_{i, k} / \sigma_{i, j}=7 / 10$, and b) profiles of the extra phase field variable $\eta_{k}$ for different ratios of $\sigma_{i, k} / \sigma_{i, j}$.

Finite values for $\eta_{k}$ at the grain boundary between grains $i$ and $j$ give also rise to extra positive contributions in the free energy, namely $(\kappa / 2)\left(\nabla \eta_{k}\right)^{2}, \gamma_{i, k} \eta_{i}^{2} \eta_{k}^{2}$ and $\gamma_{j, k} \eta_{j}^{2} \eta_{k}^{2}$. However, for a difference between $\kappa_{i, k}$ and $\kappa_{i, j}$ large enough, the decrease of the free energy due to the local reduction of the value of $\kappa$ overcomes the increase due to the extra terms in the free energy. This is illustrated in figure 12. The presence of an extra phase field variable in the diffuse grain boundary region, disturbs the original profiles for $\eta_{i}(\mathrm{x})$ and $\eta_{j}(\mathrm{x})$. The magnitude of the contribution from the third phase field variable $\eta_{k}$ depends on the relative values of the grain boundary energies $\sigma_{i, j}, \sigma_{i, j}$ and $\sigma_{j, k}$. As a result, the specific grain boundary energy and the grain boundary velocity in the simulations is lower than expected from relations (45) and (47), and the equilibrium angles between intersecting grain boundaries at triple junctions deviate from the theoretical. If more phase field variables are added to the representation, several extra contributions may appear at a grain boundary. Remark that expression (43) for the homogeneous free energy does not give rise to such a driving force for relaxation with respect to misorientation.

This local relaxation with respect to misorientation at grain boundaries is indeed relevant for diffuse boundaries. However, in the case of metals, the diffuse character of the grain boundaries is artificially introduced in phase field models to avoid tracking of the moving grain boundaries. In reality, grain boundaries in metals are atomically sharp transitions from 
one crystal orientation to another. Local relaxations at grain boundaries are on the atomistic scale and should not be treated explicitly in mesoscale grain growth simulations. In fact, their effect is already accounted for in the mesoscopic values of the specific grain boundary energy and mobility. To obtain in the phase field simulations, the grain boundary behavior observed for metals and to reproduce the parameter relations (37) exactly for individual grain boundaries and inclinations, the local values of $\kappa, \gamma$ and $L$ must be considered as fixed for a given grain configuration. The $\eta$-dependence of $\kappa$ is by consequence merely to locate and characterize the grain boundaries and must be omitted in the derivation of the driving force, giving

$$
\begin{gathered}
(1 / 2)\left(\partial \kappa / \partial \eta_{i}\right) \sum_{j=1}^{p}\left(\nabla \eta_{j}\right)^{2}=0 \\
\sum_{k=1}^{p} \frac{\partial^{2} \kappa}{\partial \eta_{k} \partial \phi_{i, j}} \nabla \eta_{k}=0
\end{gathered}
$$

and

$$
\sum_{j=1}^{p} \frac{\partial \kappa}{\partial \eta_{j}}\left(\nabla \eta_{j}\right) \cdot\left(\nabla \eta_{i}\right)=0
$$

in equations (56a), (56c) and (56d). Note that the terms (57) equal zero anyway within grains (where $\sum_{i} \sum_{j>i} \eta_{i}^{2} \eta_{j}^{2}=0$ and $\sum \nabla\left(\eta_{i}\right)^{2}=0$ ) and at grain boundaries (where $\kappa=\kappa_{i, j}\left(\phi_{i, j}\right)$ does not directly depend on the phase field variables $\eta_{i}$ ). By consequence, all results from the sharp interface asymptotic derivation in appendix A remain valid if restrictions (57) are applied. Only at junctions where 3 or more grain boundaries intersect, a finite contribution is neglected by omitting the $\eta$-dependence of $\kappa$. The results in table I and other applications of the mode ${ }^{27,49}$ show that the theoretical equilibrium angles at triple junctions are quite well reproduced, if the resolution of the numerical technique is high enough. Nevertheless, it remains to be analyzed in more detail, if and to which extent restrictions (57) affect the behavior of multi-junctions in the simulations.

A similar approach is used in other mesoscale simulation techniques for grain growth, such as Monte Carlo Potts ${ }^{10}$ and cellular automata ${ }^{50}$ simulations, to treat misorientation dependence. In these techniques, the local grain boundary energy is also determined based on the orientations of the neighboring grid points and changes ad-hoc when the orientation of one of the neighboring grid points has changed due to grain boundary movement. Local changes in the misorientation, driven by the possibility to obtain a lower value for the specific grain boundary energy, are not considered either. 
To allow a fully variational derivation of the kinetic equations, the free energy functional should be formulated in such a way that the presence of extra phase field variables at interfaces give rise to an increase of the grain boundary energy for any ratio of the parameters. This is the case for the model (3)-(4) with constant model parameters. For the model for nonuniform grain boundary properties, the contribution from extra phase field variables at grain boundaries is largely reduced when using higher powers of the phase field variables in equations (41), namely

$$
\kappa=\frac{\kappa_{i, j} \eta_{i}^{2 a} \eta_{j}^{2 a}}{\eta_{i}^{2 a} \eta_{j}^{2 a}},
$$

with $a>1$. However, ever increasing powers are required for larger ratios of $\sigma_{\max } / \sigma_{\min }$, which is impractical for the numerical solution. Other modifications to the free energy functional in a phase field model have been proposed to avoid or limit the presence of extra phase field variables at interfaces ${ }^{51,52}$. However, it seems to be extremely difficult to formulate a free energy for diffuse interface systems that avoids completely the presence of extra phases at grain boundaries for arbitrary systems (arbitrary number of orientations, arbitrarily high ratio of $\left.\sigma_{g b, \max } / \sigma_{g b, \min }\right)$, and is also applicable for numerical simulations.

\section{CONCLUSIONS}

A generalized phase field model has been formulated to perform simulations for grain growth in anisotropic systems with a high controllability of the numerical accuracy. Furthermore, an iterative algorithm is derived to calculate an appropriate set of misorientation dependent model parameters that reproduces the given grain boundary energy and mobility. Different from most phase field models, the inclination dependence of the grain boundary energy is reflected by both, the homogeneous and gradient contributions in the free energy. The proposed model formulation and model parameter choice guarantee a constant diffuse grain boundary width in order to resolve the movement of all grain boundaries with the same accuracy.

The free energy and the diffuse profiles of the phase field variables at grain boundaries have been analyzed using a combined analytical and numerical approach. Properties related to the specific grain boundary energy and diffuse interface width, were calculated numerically for a large range of values for the dimensionless parameter $\gamma$. The calculated data were interpolated using polynomials and other analytical functions. In this way, relationships 
with very high accuracy are obtained between the model parameters and grain boundary properties. The width of the diffuse grain boundaries in the phase field description is defined referring to the maximum gradient of the phase field variables, so that it can be used as a parameter in numerical criteria.

Since structural relaxations at grain boundaries are on an atomistic scale and should therefore not be considered in mesoscale simulations, the model parameters have discrete values for discrete misorientations and are assumed to be fixed for a given grain configuration. On the other hand, to describe the grain boundary reorientation due to the Herring torque, which occurs on a mesoscale, the free energy and the model parameters are differentiable with respect to inclination dependence. Various strengths and shapes of inclination dependence can be considered.

Test applications show that the grain boundary velocity expected from the GibbsThomson law, is well reproduced for $R / \ell_{g b}>4$ and $\ell_{g b} / \Delta x \approx 10$, with $\ell_{g b}$ the diffuse grain boundary width, $R$ the mean grain boundary curvature and $\Delta x$ the grid spacing. For non-uniform systems, a finer resolutions may be required to resolve triple junctions for large ratios of $\sigma_{g b, \max } / \sigma_{g b, \text { min }}$, especially for geometries where 2 low-energy boundaries meet 1 high-energy boundary.

In future work, the effect of model formulation, parameter values and numerical resolution on the behavior of triple junctions will be studied in more detail. Furthermore, expression (40) for grain boundary or interface energy can be combined with bulk free energies for different phases. In this way the model can be extended to describe phase transformations and diffusion in polycrystalline multi-phase and multi-component structures.

* Electronic address: Nele.Moelans@mtm.kuleuven. be

1 D. Fan, C. Geng, and L.-Q. Chen, Acta Mater. 45, 1115 (1997).

2 C. K. III and L.-Q. Chen, Acta Mater. 50, 3057 (2002).

3 S. Kim, D. Kim, W. Kim, and Y. Park, Phys. Rev. E 74, 061605 (2006).

4 D. Zöllner and P. Streitenberger, Scripta Mater. 54, 1697 (2006).

5 M. Miodownik, E. A. Holm, and G. N. Hassold, Scr. Mater. 42, 1173 (2000).

6 N. Moelans, B. Blanpain, and P. Wollants, Acta Mater. 54, 1175 (2006). 
7 Y. Suwa, Y. Saito, and H. Onodera, Scripta Mater. 55, 407 (2006).

8 N. Moelans, B. Blanpain, and P. Wollants, Acta Mater. 55, 2173 (2007).

9 Y. Suwa, Y. Saito, and H. Onodera, Acta Mater. 55, 6881 (2007).

10 E. Holm, G. Hassold, and M. Miodownik, Acta Mater. 49, 2981 (2001).

11 M. Upmanyu, G. Hassold, A. Kazaryan, E. Holm, Y. Wang, B. Patton, and D. Srolovitz, Interface Science 10, 201 (2002).

12 A. Kazaryan, B. Patton, S. Dregia, and Y. Wang, Acta Mater. 50, 499 (2002).

13 Y. Suwa, Y. Saito, and H. Onodera, Comp. Mater. Sci. 40, 40 (2007).

14 E. Holm, M. Miodownik, and A. Rollett, Acta Mater. 51, 2701 (2003).

15 N. Moelans, B. Blanpain, and P. Wollants, submitted for Phys. Rev. Lett.

16 L.-Q. Chen and W. Yang, Phys. Rev. B 50, 15752 (1994).

17 L.-Q. Chen, Scripta Metall. Mater. 32, 115 (1995).

18 D. Fan and L.-Q. Chen, Acta Mater. 45, 611 (1997).

19 A. Kazaryan, Y. Wang, S. Dregia, and B. Patton, Phys. Rev. B 61, 14275 (2000).

20 A. Kazarayan, Y. Wang, S. Dregia, and B. Patton, Acta Mater. 50, 2491 (2002).

21 B. Radhakrishnan, G. Sarma, T. Zacharia, A. Kazaryan, and Y. Wang, in Microstruc. Mod. Pred. Dur. Thermomech. Proc., Nov 4-8, Indianopolis, ID, Inited States (Minerals, Metals and Materials Society, 2001), pp. 47-56.

22 N. Ma, A. Kazaryan, S. Dregia, and Y. Wang, Acta Mater. 52, 3869 (2004).

23 Y. Suwa and Y. Saito, Mater. Trans. 46, 1208 (2005).

24 D. Fan and L.-Q. Chen, Phil. Mag. Lett. 75, 187 (1997).

25 D. Fan, L.-Q. Chen, and S. Chen, Mater. Sci. Eng. A 238, 78 (1997).

26 N. Ma, Q. Chen, and Y. Wang, Scripta Mater. 54, 1919 (2006).

27 N. Moelans and F. Spaepen, research in progress.

28 I. Steinbach, F. Pezzolla, B. Nestler, M. Seeßelber, R. Prieler, G. J. Schmitz, and J. L. L. Rezende, Physica D 94, 135 (1996).

29 R. Kobayashi, J. A. Warren, and W. C. Carter, Physica D 119, 415 (1998).

30 N. Moelans, B. Blanpain, and P. Wollants, Acta Mater. 53, 1771 (2005).

31 J. Cahn and J. Hilliard, J. Chem. Phys. 28, 258 (1958).

32 R. Weinstock, Calculus of variations (McGraw-Hill Book Company Inc., 1952), chap. 3, pp. $16-26$. 
34 A. Wheeler and G. McFadden, Euro. J. Appl. Math. 7, 367 (1996).

35 A. Wheeler and G. McFadden, Proc. Roy. Soc. London, A-Math. Phys. and Engn. Sci 453, 1611 (1997).

36 G. McFadden, A. Wheeler, R. Braun, and S. Coriell, Phys. Rev. E 48, 2016 (1994).

37 F. J. Humphreys and M. Hatherly, Recrystallization and related annealing phenomena (Pergamon, Oxford, 1996).

38 N. Moelans, The procedure has been implemented using Matlab. (2007).

39 J. Cahn and D. Hoffman, Acta Metall. 22, 1205 (1974).

40 C. Herring, Phys. Rev. 82 (1951).

41 J. Eggleston, G. McFadden, and P. Voorhees, Physica D 150, 91 (2001).

42 J. Debierre, A. Karma, F. Celestini, and R. Guerin, Phys. Rev. E 68, 041604 (2003).

43 I. Loginova, J. Ȧgren, and G. Amberg, Acta Mater. 52, 4055 (2004).

44 A. Karma and W.-J. Rappel, Phys. Rev. E 57, 4323 (1998).

45 T. Haxhimali, A. Karma, F. Gonzales, and M. Rappaz, Nature Materials 5, 660 (2006).

46 B. Nestler and A. Wheeler, Phys. Rev. E 57, 2602 (1998).

47 D. Hoffman and J. Cahn, Surface Sci. 31, 368 (1972).

48 S. De Groot and P. Mazur, Non-equilibrium thermodynamics (North-Holland publishing company, Amsterdam, 1963), 1st ed.

49 N. Moelans, F. Wendler, and B. Nestler, J. Comp. Phys. (in prep.).

50 K. Janssens, Model. Simul. Mater. Sci. Engn. 11, 157 (2003).

51 H. Garcke, B. Nestler, and B. Stinner, SIAM J. Appl. Math. 64, 775 (2004).

52 R. Folch and M. Plapp, Phys. Rev. E 72, 011602 (2005).

\section{APPENDIX A: ASYMPTOTIC ANALYSIS}

In this appendix, the grain boundary velocity and the force balance at edges and multijunctions are derived in the sharp interface limit for the presented model, following the procedure described in ${ }^{35,36,46}$. To be illustrative and simplify the notation, a 2-dimensional system is considered. The inclination $\phi_{i, j}=\left(\nabla \eta_{i}-\nabla \eta_{j}\right) /\left(\left|\nabla \eta_{i}-\nabla \eta_{j}\right|\right)$ then corresponds 
with a single inclination angle

$$
\phi_{i, j}^{\prime}=\arctan \left(\frac{\nabla_{y} \eta_{i}-\nabla_{y} \eta_{j}}{\nabla_{x} \eta_{i}-\nabla_{x} \eta_{j}}\right)
$$

measured with reference to the axis of the $\mathrm{x}$-coordinate. The evolution equations for a two-grain system with inclination dependent grain boundary energy are

$$
\begin{aligned}
\frac{\partial \eta_{i}}{\partial t}= & -L_{i, j}\left[m \frac{\partial f_{0}}{\partial \eta_{i}}\right. \\
- & m \nabla \cdot\left[\frac{\partial \gamma_{i, j}}{\partial \phi_{i, j}^{\prime}} \frac{\partial \phi_{i, j}^{\prime}}{\partial\left(\nabla \eta_{i}\right)} \eta_{i}^{2} \eta_{j}^{2}\right] \\
- & \nabla \cdot\left[\frac{1}{2} \frac{\partial \kappa_{i, j}}{\partial \phi_{i, j}^{\prime}} \frac{\partial \phi_{i, j}^{\prime}}{\partial\left(\nabla \eta_{i}\right)}\left(\left(\nabla \eta_{i}\right)^{2}+\left(\nabla \eta_{j}\right)^{2}\right)\right. \\
& \left.\left.+\kappa \nabla \eta_{i}\right]\right] \cdot \\
\frac{\partial \eta_{j}}{\partial t}= & -L_{i, j}\left[m \frac{\partial f_{0}}{\partial \eta_{j}}\right. \\
- & m \nabla \cdot\left[\frac{\partial \gamma_{i, j}}{\partial \phi_{i, j}^{\prime}} \frac{\partial \phi_{i, j}^{\prime}}{\partial\left(\nabla \eta_{j}\right)} \eta_{i}^{2} \eta_{j}^{2}\right] \\
- & \nabla \cdot\left[\frac{1}{2} \frac{\partial \kappa_{i, j}}{\partial \phi_{i, j}^{\prime}} \frac{\partial \phi_{i, j}^{\prime}}{\partial\left(\nabla \eta_{j}\right)}\left(\left(\nabla \eta_{i}\right)^{2}+\left(\nabla \eta_{j}\right)^{2}\right)\right. \\
& \left.\left.+\kappa \nabla \eta_{j}\right]\right],
\end{aligned}
$$

Elaboration of the separate terms in the equation for $\eta_{i}$, using the relations worked out in appendix B, gives

$$
\begin{aligned}
&- m \nabla \cdot\left[\frac{\partial \gamma_{i, j}}{\partial \phi_{i, j}^{\prime}} \frac{\partial \phi_{i, j}^{\prime}}{\partial\left(\nabla \eta_{i}\right)} \eta_{i}^{2} \eta_{j}^{2}\right] \\
&=-m\left[\frac{\partial^{2} \gamma_{i, j}}{\partial \phi_{i, j}^{\prime 2}} \frac{\eta_{i}^{2} \eta_{j}^{2}}{\left|\nabla \eta_{i}-\nabla \eta_{j}\right|^{2}}\right. \\
&\left(\nabla \phi_{i, j}^{\prime}\right) \cdot\left(\begin{array}{c}
-\left(\nabla_{y} \eta_{i}-\nabla_{y} \eta_{j}\right) \\
\nabla_{x} \eta_{i}-\nabla_{x} \eta_{j}
\end{array}\right) \\
&\left.+\frac{\partial \gamma_{i, j}}{\partial \phi_{i, j}^{\prime}}\left(\begin{array}{c}
-\left(\nabla_{y} \eta_{i}-\nabla_{y} \eta_{j}\right) \\
\nabla
\end{array}\right) \cdot \nabla \frac{\eta_{i}^{2} \eta_{j}^{2}}{\left|\nabla \eta_{i}-\nabla \eta_{j}\right|^{2}}\right] \\
&=-m\left[\frac{\partial^{2} \gamma_{i, j}}{\partial \phi_{i, j}^{\prime 2}} \frac{\eta_{i}^{2} \eta_{j}^{2}}{\left|\nabla \eta_{i}-\nabla \eta_{j}\right|} \nabla \cdot \frac{\nabla \eta_{i}-\nabla \eta_{j}}{\left|\nabla \eta_{i}-\nabla \eta_{j}\right|}\right]
\end{aligned}
$$




$$
\begin{aligned}
& -\nabla \cdot\left[\frac{1}{2} \frac{\partial \kappa_{i, j}}{\partial \phi_{i, j}^{\prime}} \frac{\partial \phi_{i, j}^{\prime}}{\partial\left(\nabla \eta_{i}\right)}\left(\left(\nabla \eta_{i}\right)^{2}+\left(\nabla \eta_{j}\right)^{2}\right)\right] \\
& =-\left[\frac{1}{2} \frac{\partial^{2} \kappa_{i, j}}{\partial \phi_{i, j}^{\prime 2}} \frac{\left(\nabla \eta_{i}\right)^{2}+\left(\nabla \eta_{j}\right)^{2}}{\left|\nabla \eta_{i}-\nabla \eta_{j}\right|^{2}}\right. \\
& \left(\nabla \phi_{i, j}^{\prime}\right) \cdot\left(\begin{array}{c}
-\left(\nabla_{y} \eta_{i}-\nabla_{y} \eta_{j}\right) \\
\nabla_{x} \eta_{i}-\nabla_{x} \eta_{j}
\end{array}\right) \\
& \left.+\frac{\partial \kappa_{i, j}}{\partial \phi_{i, j}^{\prime}}\left(\begin{array}{c}
-\left(\nabla_{y} \eta_{i}-\nabla_{y} \eta_{j}\right) \\
\nabla_{x} \eta_{i}-\nabla_{x} \eta_{j}
\end{array}\right) \cdot \nabla \frac{\left(\nabla \eta_{i}\right)^{2}+\left(\nabla \eta_{j}\right)^{2}}{\left|\nabla \eta_{i}-\nabla \eta_{j}\right|^{2}}\right] \\
& =-\frac{1}{2} \frac{\partial^{2} \kappa_{i, j}}{\partial \phi_{i, j}^{\prime 2}} \frac{\left(\nabla \eta_{i}\right)^{2}+\left(\nabla \eta_{j}\right)^{2}}{\left|\nabla \eta_{i}-\nabla \eta_{j}\right|^{2}} \\
& \left|\nabla \eta_{i}-\nabla \eta_{j}\right| \nabla \cdot \frac{\nabla \eta_{i}-\nabla \eta_{j}}{\left|\nabla \eta_{i}-\nabla \eta_{j}\right|}
\end{aligned}
$$

and

$$
-\nabla \cdot\left[\kappa \nabla \eta_{i}\right]=-\frac{\partial \kappa_{i, j}}{\partial \phi_{i, j}^{\prime}} \nabla \phi_{i, j}^{\prime} \cdot \nabla \eta_{i}-\kappa \nabla^{2} \eta_{i}
$$

Analogous expressions are obtained for $\eta_{j}$, except that equations (A3) and (A4) change sign because of the antisymmetric formulation of $\phi_{i, j}^{\prime}$ with respect to $\eta_{i}$ and $\eta_{j}$.

If $\kappa_{i, j}\left(\phi_{i, j}^{\prime}\right)$ is replaced by $\left(k_{i, j}\left(\phi_{i, j}^{\prime}\right)\right)^{2}$, and accordingly $\partial \kappa_{i, j} / \partial \phi_{i, j}^{\prime}$ by $2 k_{i, j} \partial k_{i, j} / \partial \phi_{i, j}^{\prime}$ and $\partial^{2} \kappa_{i, j} / \partial \phi_{i, j}^{\prime 2}$ by $2\left(\left(\partial k_{i, j} / \partial \phi_{i, j}\right)^{\prime 2}+k_{i, j} \partial^{2} k_{i, j} / \partial \phi_{i, j}^{\prime 2}\right)$, expressions (A4) and (A5) resemble the terms in equation 27 of Ref. ${ }^{36}$. More specifically, expression (A5) corresponds with the first and second term on the right hand side and expression (A4) with the third term. The contributions from these terms to the leading and first order solutions of the phase field equations are thus taken from Ref. ${ }^{36}$. Contribution (A3), which comes from the inclination dependence of the homogeneous free energy, is not present in the evolution equation for the model discussed in Ref. ${ }^{36}$. Applying the asymptotic expansions 55-58 from Ref. ${ }^{36}$, it can be verified that the effects from contribution (A3) on the solution of the phase field equations are of first and higher order in $\epsilon$, with $\epsilon$ a measure for the width of the diffuse interface as defined in $\operatorname{Ref}^{36}$.

Comparison of the different terms in (A4) and (A5) with those on the RH side of equation 27 of Ref. ${ }^{36}$ and following the procedure described in Ref. ${ }^{36}$, gives that the phase field profiles $\eta_{i}(r)$ and $\eta_{j}(r)$ across a diffuse grain boundary satisfy to leading order equations (7) and (8) derived for a flat grain boundary, with $\kappa_{i, j}=\kappa_{i, j}\left(\phi_{i, j}^{\prime}\right)$ and $\gamma_{i, j}=\gamma_{i, j}\left(\phi_{i, j}^{\prime}\right)$ constant and $x$ replaced by $r$, the coordinate along the normal to the grain boundary surface. To obtain the 
sharp interface approximation (for $\ell_{g b} \ll R_{1}, R_{2}$ or $\epsilon \rightarrow 0$ ) of the grain boundary velocity, it is first assumed that the inclination dependence of the homogeneous free energy density $m f_{0}$ has a form

$$
\begin{aligned}
m f_{0}\left(\gamma_{i, j}\left(\phi_{i, j}^{\prime}\right)\right) & =m_{i, j}^{\prime}\left(\gamma_{i, j}\left(\phi_{i, j}^{\prime}\right)\right) f_{0}\left(\bar{\gamma}_{i, j}\right) \\
& =m_{i, j}^{\prime}\left(\phi_{i, j}^{\prime}\right) f_{0}\left(\bar{\gamma}_{i, j}\right)
\end{aligned}
$$

where $m_{i, j}^{\prime}\left(\phi_{i, j}^{\prime}\right)$ has the same inclination dependent factor as $\kappa_{i, j}$, for instance,

$$
m_{i, j}^{\prime}=\bar{m}_{i, j}^{\prime} f_{\text {incl }}\left(\phi_{i, j}^{\prime}\right) \quad \text { for } \quad \kappa_{i, j}=\bar{\kappa}_{i, j} f_{\text {incl }}\left(\phi_{i, j}^{\prime}\right)
$$

The contribution in the driving force associated with the inclination dependence of the homogeneous free energy can then be formulated as

$$
\begin{array}{r}
-\nabla \cdot \frac{\partial\left(m f_{0}\left(\gamma_{i, j}\left(\phi_{i, j}^{\prime}\right)\right)\right)}{\partial\left(\nabla \eta_{i}\right)}=-\nabla \cdot \frac{\partial\left(m_{i, j}^{\prime} f_{0}\left(\bar{\gamma}_{i, j}\right)\right)}{\partial\left(\nabla \eta_{i}\right)} \\
=-\frac{\partial^{2} m_{i, j}^{\prime}}{\partial \phi_{i, j}^{\prime 2}} \frac{f_{0}\left(\bar{\gamma}_{i, j}\right)}{\left|\nabla \eta_{i}-\nabla \eta_{j}\right|^{2}} \\
\left(\nabla \phi_{i, j}^{\prime}\right) \cdot\left(\begin{array}{c}
-\left(\nabla_{y} \eta_{i}-\nabla_{y} \eta_{j}\right) \\
\nabla_{x} \eta_{i}-\nabla_{x} \eta_{j}
\end{array}\right) \\
=\frac{1}{2} \frac{\partial^{2} \kappa_{i, j}^{\prime}}{\partial \phi_{i, j}^{\prime 2}} \frac{\left(\nabla \eta_{i}\right)^{2}+\left(\nabla \eta_{j}\right)^{2}}{\left|\nabla \eta_{i}-\nabla \eta_{j}\right|^{2}} \\
\left(\nabla \phi_{i, j}^{\prime}\right) \cdot\left(\begin{array}{c}
-\left(\nabla_{y} \eta_{i}-\nabla_{y} \eta_{j}\right) \\
\nabla_{x} \eta_{i}-\nabla_{x} \eta_{j}
\end{array}\right)
\end{array}
$$

where the leading order approximation (8) for the phase field profiles along direction $\phi_{i, j}^{\prime}$ is used to obtain (A9) from (A8). Expression (A9) is exactly the same as that obtained in (A4). Summation of (A4), (A9) and (A5) gives

$$
\begin{aligned}
&-\nabla \cdot\left[\frac{\partial f}{\partial\left(\nabla \eta_{i}\right)}\right]=-\frac{\partial^{2} \kappa_{i, j}}{\partial \phi_{i, j}^{\prime 2}} \frac{\left(\nabla \eta_{i}\right)^{2}+\left(\nabla \eta_{j}\right)^{2}}{\left|\nabla \eta_{i}-\nabla \eta_{j}\right|^{2}} \\
&\left|\nabla \eta_{i}-\nabla \eta_{j}\right| \nabla \cdot \frac{\nabla \eta_{i}-\nabla \eta_{j}}{\left|\nabla \eta_{i}-\nabla \eta_{j}\right|} \\
&-\frac{\partial \kappa_{i, j}}{\partial \phi_{i, j}^{\prime}} \nabla \phi_{i, j}^{\prime} \cdot \nabla \eta_{i}-\kappa \nabla^{2} \eta_{i},
\end{aligned}
$$

with $f$ the free energy density. Each of the terms corresponds with a term in expression 27 in Ref. ${ }^{36}$, except that there is a dependence of 2 phase field variables. Using the asymptotic 
expansions 55-58 from Ref. ${ }^{36}$ and considering that $\left(\nabla \eta_{i}-\nabla \eta_{j}\right) /\left|\nabla \eta_{i}-\nabla \eta_{j}\right|=\left(\nabla \eta_{i}\right) /\left|\nabla \eta_{i}\right|=$ $\left(-\nabla \eta_{j}\right) /\left|\nabla \eta_{j}\right|$, the solvability condition (equation 70 in Ref. ${ }^{36}$ ) for $\eta_{i}$ states that

$$
\begin{aligned}
\frac{v_{g b, n}}{L} \int_{0}^{+\infty}\left(\frac{\partial \eta_{i}}{\partial r}\right)^{2} \mathrm{~d} r= \\
-\frac{\partial^{2} \kappa_{i, j}}{\partial \phi_{i, j}^{\prime 2}} \frac{1}{R} \int_{0}^{+\infty}\left[\frac{\left(\frac{\partial \eta_{i}}{\partial r}\right)^{2}+\left(\frac{\partial \eta_{j}}{\partial r}\right)^{2}}{\left(\frac{\partial \eta_{i}}{\partial r}\right)-\left(\frac{\partial \eta_{j}}{\partial r}\right)}\right] \frac{\partial \eta_{i}}{\partial r} \mathrm{~d} r \\
-\frac{\partial \kappa_{i, j}}{\partial \phi_{i, j}^{\prime}} \frac{\partial}{\partial t} \int_{0}^{+\infty}\left(\frac{\partial \eta_{i}}{\partial r}\right)^{2} \mathrm{~d} r \\
-\frac{\kappa}{R} \int_{0}^{+\infty}\left(\frac{\partial \eta_{i}}{\partial r}\right)^{2} \mathrm{~d} r
\end{aligned}
$$

with $v_{g b, n}$ as defined in equation (33). An analogous equation is obtained for the solvability condition for $\eta_{j}$, except that the first term on the RH side changes sign. Summation of equation (A11) for the solvability condition for $\eta_{i}$ with the solvability condition for $\eta_{j}$ and rearrangement of the terms, then gives

$$
v_{g b, n}=-\frac{L \bar{\kappa}_{i, j}}{R}\left(f_{\mathrm{incl}}+\frac{\partial^{2} f_{\text {incl }}}{\partial \phi_{i, j}^{\prime 2}}\right),
$$

for the normal velocity of a point on a grain boundary surface.

Following the procedure described in ${ }^{35,46}$, the force of a diffuse grain boundary acting on a plane that cuts its surface, can be calculated in the sharp interface limit. Assume, for instance, a grain boundary surface with normal $\mathbf{r}$ as in figure $1 \mathrm{~b}$ and a plane cutting the surface oriented perpendicular to it, with normal $\mathbf{t}_{1}$ and so that direction $\mathbf{t}_{2}$ is common to the grain boundary surface and the plane. The local force acting on the plane is given by

$$
F=\Xi \cdot \mathbf{t}_{1}
$$

with $\Xi$ a tensor with, for the current model, the elements

$$
\Xi_{p q}=\nabla_{p} \eta_{i} \frac{\partial f}{\partial\left(\nabla_{q} \eta_{i}\right)}+\nabla_{p} \eta_{j} \frac{\partial f}{\partial\left(\nabla_{q} \eta_{j}\right)}-\delta_{p q} f
$$

where $p, q$ run over the spatial coordinates, or in tensor notation

$$
\Xi=\nabla \eta_{i} \otimes \frac{\partial f}{\partial \nabla \eta_{i}}+\nabla \eta_{j} \otimes \frac{\partial f}{\partial \nabla \eta_{j}}-f \mathbf{I}
$$

with I the unit tensor. Following the same reasoning as for (A10) it can be obtained that for $\ell_{g b} \ll R_{1}$ and $R_{2}$ infinite

$$
\frac{\partial f}{\partial\left(\nabla \eta_{i}\right)}=\frac{\partial \kappa_{i, j}}{\partial \alpha_{1}} \frac{\partial \eta_{i}}{\partial r} \mathbf{t}_{1}+\kappa_{i, j} \frac{\partial \eta_{i}}{\partial r} \mathbf{r}
$$


with the angle $\alpha_{1}$ measured in the curvilinear coordinate system. By consequence,

$$
\Xi=\left(\left(\frac{\partial \eta_{i}}{\partial r}\right)^{2}+\left(\frac{\partial \eta_{j}}{\partial r}\right)^{2}\right)\left(\mathbf{r} \otimes\left[\frac{\partial \kappa_{i, j}}{\partial \alpha_{1}} \mathbf{t}_{1}+\kappa_{i, j} \mathbf{r}\right]-\kappa_{i, j} \mathbf{I}\right)
$$

and

$$
F=\Xi \cdot \mathbf{t}_{1}=\left(\left(\frac{\partial \eta_{i}}{\partial r}\right)^{2}+\left(\frac{\partial \eta_{j}}{\partial r}\right)^{2}\right)\left(\frac{\partial \kappa_{i, j}}{\partial \alpha_{1}} \mathbf{r}-\kappa_{i, j} \mathbf{t}_{1}\right)
$$

Integration along the phase field profiles across a diffuse grain boundary, gives the total force acting on the plane per unit length (with the length measured along direction $\mathbf{t}_{2}$ ):

$$
\begin{aligned}
f & =\int_{0}^{\infty} F \mathrm{~d} r \\
& =\int_{0}^{\infty}\left(\left(\frac{\partial \eta_{i}}{\partial r}\right)^{2}+\left(\frac{\partial \eta_{j}}{\partial r}\right)^{2}\right) \mathrm{d} r\left(\frac{\partial \kappa_{i, j}}{\partial \alpha_{1}} \mathbf{r}-\kappa_{i, j} \mathbf{t}_{1}\right) \\
& =\frac{\sigma_{g b}}{\kappa_{i, j}} \frac{\partial \kappa_{i, j}}{\partial \alpha_{1}} \mathbf{r}-\sigma_{g b} \mathbf{t}_{1},
\end{aligned}
$$

where relations (8) and (10) are used to evaluate the integral.

\section{APPENDIX B: VECTOR RELATIONS}

Consider the angle

$$
\phi_{i, j}^{\prime}=\arctan \left(\frac{\nabla_{y} \eta_{i}-\nabla_{y} \eta_{j}}{\nabla_{x} \eta_{i}-\nabla_{x} \eta_{j}}\right),
$$

measured with reference to the axis of the $\mathrm{x}$-coordinate. It can then be verified that (see also Ref. ${ }^{36}$ )

$$
\begin{aligned}
\frac{\partial \phi_{i, j}^{\prime}}{\partial \nabla \eta_{i}} & =-\frac{\partial \phi_{i, j}^{\prime}}{\partial \nabla \eta_{j}} \\
& =\frac{1}{\left|\nabla \eta_{i}-\nabla \eta_{j}\right|^{2}}\left(\begin{array}{c}
-\left(\nabla_{y} \eta_{i}-\nabla_{y} \eta_{j}\right) \\
\nabla_{x} \eta_{i}-\nabla_{x} \eta_{j}
\end{array}\right),
\end{aligned}
$$


is a vector tangential to the surface,

$$
\begin{aligned}
& -\left(\nabla \eta_{i}-\nabla \eta_{j}\right) \cdot \frac{\partial \phi_{i, j}^{\prime}}{\partial\left(\nabla \eta_{i}\right)}= \\
& -\frac{1}{\left.\mid \nabla \eta_{i}-\nabla \eta_{j}\right)\left.\right|^{2}}[ \\
& \left(\nabla_{x} \eta_{i}-\nabla_{x} \eta_{j} \nabla_{y} \eta_{i}-\nabla_{y} \eta_{j}\right) \cdot \\
& \left.\left(\begin{array}{c}
-\left(\nabla_{y} \eta_{i}-\nabla_{y} \eta_{j}\right) \\
\nabla_{x} \eta_{i}-\nabla_{x} \eta_{j}
\end{array}\right)\right]=0, \\
& \nabla \cdot\left[\left|\nabla \eta_{i}-\nabla \eta_{j}\right|^{2} \frac{\partial \phi_{i, j}^{\prime}}{\partial\left(\nabla \eta_{i}\right)}\right]= \\
& \nabla \cdot\left[\left|\nabla \eta_{i}-\nabla \eta_{j}\right|^{2} \frac{\partial \phi_{i, j}^{\prime}}{\partial\left(\nabla \eta_{j}\right)}\right]=0 \text {, }
\end{aligned}
$$

and

$$
\begin{aligned}
& \left(\nabla \phi_{i, j}^{\prime}\right) \cdot\left(\begin{array}{c}
-\left(\nabla_{y} \eta_{i}-\nabla_{y} \eta_{j}\right) \\
\nabla_{x} \eta_{i}-\nabla_{x} \eta_{j}
\end{array}\right) \\
= & \left|\nabla \eta_{i}-\nabla \eta_{j}\right| \nabla \cdot \frac{\nabla \eta_{i}-\nabla \eta_{j}}{\left|\nabla \eta_{i}-\nabla \eta_{j}\right|} .
\end{aligned}
$$

Moreover, with $\mathbf{r}$ the normal to the grain boundary surface,

$$
\begin{aligned}
\nabla\left(\nabla \eta_{i}\right)^{2} & =\nabla\left(\frac{\partial \eta_{i}}{\partial r} \mathbf{r}\right)^{2}=2\left(\frac{\partial \eta_{i}}{\partial r} \mathbf{r}\right)\left(\nabla \cdot\left(\frac{\partial \eta_{i}}{\partial r} \mathbf{r}\right)\right) \\
& =2\left(\nabla \eta_{i}\right)\left(\nabla^{2} \eta_{i}\right)
\end{aligned}
$$

and

$$
\nabla\left[\frac{1}{\left|\nabla \eta_{i}-\nabla \eta_{j}\right|}\right]=\left(\frac{\nabla \eta_{i}-\nabla \eta_{j}}{\left|\nabla \eta_{i}-\nabla \eta_{j}\right|}\right)\left(\nabla^{2} \eta_{i}-\nabla^{2} \eta_{j}\right)
$$

are directed along $\mathbf{r}$. It follows from (B6) and (B7) that the vector $\nabla\left[\left(\left(\nabla \eta_{i}\right)^{2}+\left(\nabla \eta_{j}\right)^{2}\right) / \mid \nabla \eta_{i}-\right.$ $\left.\left.\nabla \eta_{j}\right|^{2}\right]$ is directed along $\mathbf{r}$ as well. 